\title{
Electron-Positron Pair Production in Space- or Time-Dependent Electric Fields
}

\author{
Hagen Kleinert ${ }^{(a, b)}$, Remo Ruffini ${ }^{(b)}$ and She-Sheng Xue ${ }^{(b)}$ \\ (a) Institut für Theoretische Physik, Freie Universität Berlin, 14195 Berlin, Germany and \\ (b) ICRANeT Piazzale della Repubblica, 10 -65122, Pescara, and \\ Dipartimento di Fisica, University of Rome "La Sapienza", P.le A. Moro 5, 00185 Rome, Italy
}

(Dated: Received version October 27, 2018)

\begin{abstract}
Treating the production of electron and positron pairs by a strong electric field from the vacuum as a quantum tunneling process we derive, in semiclassical approximation, a general expression for the pair production rate in a $z$-dependent electric field $E(z)$ pointing in the $z$-direction. We also allow for a smoothly varying magnetic field parallel to $E(z)$. The result is applied to a confined field $E(z) \neq 0$ for $|z| \lesssim \ell$, a semi-confined field $E(z) \neq 0$ for $z \gtrsim 0$, and a linearly increasing field $E(z) \sim z$. The boundary effects of the confined fields on pair-production rates are exhibited. A simple variable change in all formulas leads to results for electric fields depending on time rather than space.

In addition, we discuss tunneling processes in which empty atomic bound states are spontaneously filled by negative-energy electrons from the vacuum under positron emission. In particular, we calculate the rate at which the atomic levels of a bare nucleus of finite size $r_{\mathrm{n}}$ and large $Z \gg 1$ are filled by spontaneous pair creation.

PACS numbers: 12.20.-m, 13.40-f, 11.27.+d, 12.20.Ds
\end{abstract}

\section{INTRODUCTION}

The creation of electron-positron pairs from the vacuum by an external uniform electric field in spacetime was first studied by Sauter [1] as a quantum tunneling process. Heisenberg and Euler [2] extended his result by calculating an effective Lagrangian from the Dirac theory for electrons in a constant electromagnetic field. A more elegant quantum field theoretic reformulation was given by Schwinger [3] based on calculations of who calculated within Quantum Electrodynamics (QED) the one-loop effective action in a constant electromagnetic field. A detailed review and relevant references can be found in Refs. [4] and [5].

Apart from its purely theoretic interest, the pair-production in nonuniform fields is experimentally relevant in collisions of laser beams [6] and heavy ions [7, 8], and as a possible explanation of the powerful Gamma Ray Bursts in astrophysics [5, 9]. It is also important for understanding the plasma oscillations [10] of electrons and positrons after their creation in electric fields.

The rate of pair-production may be split into an exponential and a pre-exponential factor. The 
exponent is determined by the classical trajectory of the tunneling particle in imaginary time which has the smallest action. It plays the same role as the activation energy in a Boltzmann factor with a "temperature" $\hbar$. The pre-exponential factor is determined by the quantum fluctuations of the path around that trajectory. At the semiclassical level, the latter is obtained from the functional determinant of the quadratic fluctuations. It can be calculated in closed form only for a few classical paths [11]. An efficient technique for doing this is based on the WKB wave functions, another on solving the Heisenberg equations of motion for the position operator in the external field [11].

Given the difficulties in calculating the pre-exponential factor, only a few nonuniform electric fields in space or in time have led to analytic results for the pair-production rate: (i) the electric field in the $z$-direction is confined in the space $x<x_{0}$, i.e., $\mathbf{E}=E(x) \hat{\mathbf{z}}$ where $E(x)=E_{0} \theta\left(x_{0}-x\right)$ [12]; (ii) the electric field in the $z$-direction depends only on the light-cone coordinate $z_{+}=(t+z) / \sqrt{2}$, i.e., $\mathbf{E}=E\left(z_{+}\right) \hat{\mathbf{z}}$ [13]. If the nonuniform field has the form $E(z)=E_{0} / \cosh ^{2}(z)$, which we shall refer to as a Sauter field, the rate was calculated by solving the Dirac equation [14] in the same way as Heisenberg and Euler did for the constant electric field. For general space and time dependences, only the exponential factor can be written down easily - the fluctuation factor is usually hard to calculate [7]. In the Coulomb field of heavy nucleus whose size is finite and charge $Z$ is supercritical, the problem becomes even more difficult for bound states being involved in pair production, and a lot of effort has been spent on this issue [7, 8, 15].

If the electric field has only a time dependence $E=E(t)$, both exponential and pre-exponential factors were approximately computed by Brezin and Itzykson using WKB methods for the purely periodic field $E(t)=E_{0} \cos \omega_{0} t$ [16]. The result was generalized by Popov in Ref. [17] to more general time-dependent fields $E(t)$. After this, several time-independent but space-dependent fields were treated, for instance an electric field between two conducting plates [18], and an electric field around a Reissner-Nordström black hole [19].

The semicalssical expansion was carried beyond the WKB approximation by calculating higherorder corrections in powers of $\hbar$ in Refs. [20] and [21]. Unfortunately, these terms do not comprise all corrections of the same orders $\hbar$ as will be explained at the end of Section $\amalg$.

An alternative approach to the same problems was recently proposed by using the worldline formalism [22], sometimes called the "string-inspired formalism". This formalism is closely related to Schwinger's quantum field theoretic treatment of the tunneling problem, where the evaluation of a fluctuation determinant is required involving the fields of the particle pairs created from the vacuum. The worldline approach is special technique for calculating precisely this functional determinant. Within the worldline formalism, Dunne and Schubert [23] calculated the exponential 
factor and Dunne et al. 24] gave the associated prefactor for various field configurations: for instance a spatially uniform, and single-pulse field with a temporal Sauter shape $\propto 1 / \cosh ^{2} \omega t$. For general $z$-dependences, a numerical calculation scheme was proposed in Ref. [25] and applied further in [26]. For a multidimensional extension of the techniques see Ref. [27].

In this article we derive a general expression for the pair-production rate in nonuniform electric fields $E(z)$ pointing in the $z$-direction and varying only along this direction. A simple variable change in all formulas leads to results for electric fields depending on time rather than space. As examples, we shall treat three cases: (i) a nonzero electric field confined to a region of size $\ell$, i.e., $E(z) \neq 0,|z| \lesssim \ell$ (Sauter field see Eq. (56) ); (ii) a nonzero electric field in a half-space, i.e., $E(z) \neq 0, z \gtrsim 0$ (see Eq. (77)); (iii) an electric field increasing linearly like $E(z) \sim z$. In addition we shall study the process of negative-energy electrons tunneling into the bound states of an electric potential with the emission of positrons. We consider two cases: (1) the electric field $E(z) \sim z$ of harmonic potential $V(z) \sim z^{2} ;(2)$ the radial Coulomb field $E(r)=e Z / r^{2}$ with large $Z$ outside the nuclear radius $r_{\mathrm{n}}$.

\section{SEMI-CLASSICAL DESCRIPTION OF PAIR PRODUCTION}

The phenomenon of pair production in an external electric field can be understood as a quantummechanical tunneling process of Dirac electrons [28, 29]. In the original Dirac picture, the electric field bends the positive and negative-energy levels of the Hamiltonian, leading to a level-crossing and a tunneling of the electrons in the negative-energy band to the positive-energy band. Let the field vector $\mathbf{E}(z)$ point in the $z$-direction. In the one-dimensional potential energy

$$
V(z)=-e A_{0}(z)=e \int^{z} d z^{\prime} E\left(z^{\prime}\right)
$$

of an electron of charge $-e$, the classical positive and negative-energy spectra are

$$
\mathcal{E}_{ \pm}\left(p_{z}, p_{\perp} ; z\right)= \pm \sqrt{\left(c p_{z}\right)^{2}+c^{2} p_{\perp}^{2}+\left(m_{e} c^{2}\right)^{2}}+V(z),
$$

where $p_{z}$ is the momentum in the $z$-direction, $\mathbf{p}_{\perp}$ the momentum orthogonal to it, and $p_{\perp} \equiv\left|\mathbf{p}_{\perp}\right|$. For a given energy $\mathcal{E}$, the tunneling takes place from $z_{-}$to $z_{+}$determined by $p_{z}=0$ in Eq. (2)

$$
\mathcal{E}=\mathcal{E}_{+}\left(0, p_{\perp} ; z_{+}\right)=\mathcal{E}_{-}\left(0, p_{\perp} ; z_{-}\right)
$$

The points $z_{ \pm}$are the turning points of the classical trajectories crossing from the positive-energy band to the negative one at energy $\mathcal{E}$. They satisfy the equations

$$
V\left(z_{ \pm}\right)=\mp \sqrt{c^{2} p_{\perp}^{2}+m_{e}^{2} c^{4}}+\mathcal{E} .
$$


This energy-level crossing $\mathcal{E}$ is shown in Fig. 1 for the Sauter potential $V(z) \propto \tanh (z / \ell)$.

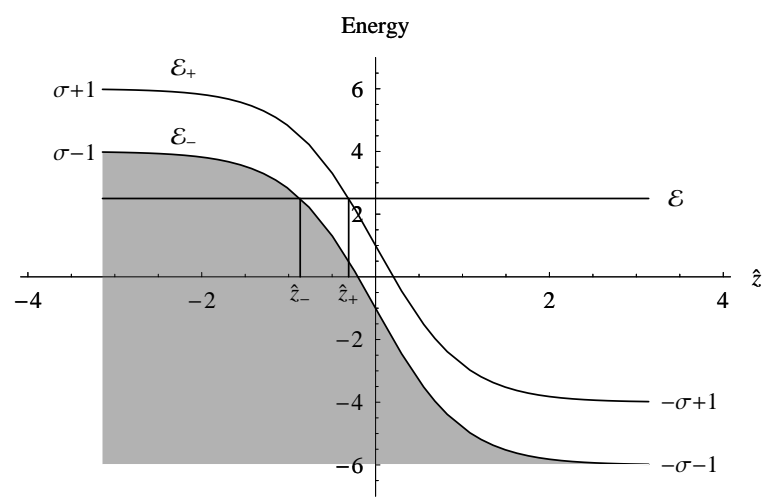

FIG. 1: Positive- and negative-energy spectra $\mathcal{E}_{ \pm}(z)$ of Eq. (2) in units of $m_{e} c^{2}$, with $p_{z}=p_{\perp}=0$ as a function of $\hat{z}=z / \ell$ for the Sauter potential $V_{ \pm}(z)$ (56) for $\sigma=5$.

\section{A. WKB transmission probability for Klein-Gordon Field}

The probability of quantum tunneling in the $z$-direction is most easily studied for a scalar field which satisfies the Klein-Gordon equation

$$
\left\{\left[i \hbar \partial_{\mu}+\frac{e}{c} A_{\mu}(z)\right]^{2}-m_{e}^{2} c^{2}\right\} \phi(x)=0,
$$

where $x_{0} \equiv c t$. If there is only an electric field in the $z$-direction which varies only along $z$, we can choose a vector potential with the only nonzero component (11), and make the ansatz $\phi(x)=e^{-i \mathcal{E} t / \hbar} e^{i \mathbf{p}_{\perp} \mathbf{x}_{\perp} / \hbar} \phi_{\mathbf{p}_{\perp}, \mathcal{E}}(z)$, with a fixed momentum $\mathbf{p}_{\perp}$ in the $x, y$ direction and an energy $\mathcal{E}$, and Eq. (5) becomes simply

$$
\left[-\hbar^{2} \frac{d^{2}}{d z^{2}}+p_{\perp}^{2}+m_{e}^{2} c^{2}-\frac{1}{c^{2}}[\mathcal{E}-V(z)]^{2}\right] \phi_{\mathbf{p}_{\perp}, \mathcal{E}}(z)=0 .
$$

By expressing the wave function $\phi_{\mathbf{p}_{\perp}, \mathcal{E}}(z)$ as an exponential

$$
\phi_{\mathbf{p}_{\perp}, \mathcal{E}}(z)=\mathcal{C} e^{i S_{\mathbf{p}_{\perp}, \mathcal{E}} / \hbar}
$$

where $\mathcal{C}$ is some normalization constant, the wave equation becomes a Riccatti equation for $S_{\mathbf{p}_{\perp}, \mathcal{E}}$ :

$$
-i \hbar \partial_{z}^{2} S_{\mathbf{p}_{\perp}, \mathcal{E}}(z)+\left[\partial_{z} S_{\mathbf{p}_{\perp}, \mathcal{E}}(z)\right]^{2}-p_{z}^{2}(z)=0
$$


where the function $p_{z}(z)$ is the solution of the equation

$$
p_{z}^{2}(z)=\frac{1}{c^{2}}[\mathcal{E}-V(z)]^{2}-p_{\perp}^{2}-m_{e}^{2} c^{2}
$$

The solution of Eq. (8) can be found iteratively as an expansion in powers of $\hbar$ :

$$
S_{\mathbf{p}_{\perp}, \mathcal{E}}(z)=S_{\mathbf{p}_{\perp}, \mathcal{E}}^{(0)}(z)-i \hbar S_{\mathbf{p}_{\perp}, \mathcal{E}}^{(1)}(z)+(-i \hbar)^{2} S_{\mathbf{p}_{\perp}, \mathcal{E}}^{(2)}(z)+\ldots
$$

Neglecting the expansion terms after $S_{\mathbf{p}_{\perp}, \mathcal{E}}^{(1)}(z)=-\log p_{z}^{1 / 2}(z)$ leads to the WKB approximation for the wave functions of positive and negative energies can (see e.g. [30, 31])

$$
\phi_{\mathbf{p}_{\perp}, \mathcal{E}}^{\mathrm{WKB}}(z)=\frac{\mathcal{C}}{p_{z}^{1 / 2}(z)} e^{i S_{\mathbf{p}_{\perp}, \mathcal{E}}^{(0)}(z) / \hbar}
$$

where $S_{\mathbf{p}_{\perp}, \mathcal{E}}^{(0)}(z)$ is the eikonal

$$
S_{\mathbf{p}_{\perp}, \mathcal{E}}^{(0)}(z)=\int^{z} p_{z}\left(z^{\prime}\right) d z^{\prime}
$$

Between the turning points $z_{-}<z<z_{+}$, whose positions are illustrated in Fig. 1, the momentum $p_{z}(z)$ is imaginary and is useful to define the positive function

$$
\kappa_{z}(z) \equiv \sqrt{p_{\perp}^{2}+m_{e}^{2} c^{2}-\frac{1}{c^{2}}[\mathcal{E}-V(z)]^{2}} \geq 0 .
$$

The tunneling wave function in this regime is the linear combination

$$
\frac{\mathcal{C}}{2\left(\kappa_{z}\right)^{1 / 2}} \exp \left[-\frac{1}{\hbar} \int_{z_{-}}^{z} \kappa_{z} d z\right]+\frac{\overline{\mathcal{C}}}{2\left(\kappa_{z}\right)^{1 / 2}} \exp \left[+\frac{1}{\hbar} \int_{z_{-}}^{z} \kappa_{z} d z\right] .
$$

Outside the turning points, i.e., for $z<z_{-}$and $z>z_{+}$, there exist negative-energy and positiveenergy solutions for $\mathcal{E}<\mathcal{E}_{-}$and $\mathcal{E}>\mathcal{E}_{+}$for positive $p_{z}$. On the left-hand side of $z_{-}$, the general solution is a linear combination of an incoming wave running to the right and outgoing wave running to the left:

$$
\frac{\mathcal{C}_{+}}{\left(p_{z}\right)^{1 / 2}} \exp \left[\frac{i}{\hbar} \int^{z} p_{z} d z\right]+\frac{\mathcal{C}_{-}}{\left(p_{z}\right)^{1 / 2}} \exp \left[-\frac{i}{\hbar} \int^{z} p_{z} d z\right]
$$

On the right hand of $z_{+}$, there is only an outgoing wave

$$
\frac{\mathcal{T}}{\left(p_{z}\right)^{1 / 2}} \exp \left[\frac{i}{\hbar} \int_{z_{+}}^{z} p_{z} d z\right]
$$

The connection equations can be solved by

$$
\overline{\mathcal{C}}=0, \quad \mathcal{C}_{ \pm}=e^{ \pm i \pi / 4} \mathcal{C} / 2, \quad \mathcal{T}=\mathcal{C}_{+} \exp \left[-\frac{1}{\hbar} \int_{z_{-}}^{z_{+}} \kappa_{z} d z\right]
$$


The incident flux density is

$$
j_{z} \equiv \frac{\hbar}{2 m_{e} i}\left[\phi^{*} \partial_{z} \phi-\left(\partial_{z} \phi^{*}\right) \phi\right]=\frac{p_{z}}{m_{e}} \phi^{*} \phi=\frac{\left|\mathcal{C}_{+}\right|^{2}}{m_{e}},
$$

which can be written as

$$
j_{z}(z)=v_{z}(z) n_{-}(z)
$$

where $v_{z}(z)=p_{z}(z) / m_{e}$ is the velocity and $n_{-}(z)=\phi^{*}(z) \phi(z)$ the density of the incoming particles. Note that the $z$-dependence of $v_{z}(z)$ and $n_{-}(z)$ cancel each other. By analogy, the outgoing flux density is $|\mathcal{T}|^{2} / m_{e}$.

\section{B. Rate of pair production}

From the above considerations we obtain for the transmission probability

$$
W_{\mathrm{WKB}} \equiv \frac{\text { transmitted flux }}{\text { incident flux }}
$$

the simple exponential

$$
W_{\mathrm{WKB}}\left(p_{\perp}, \mathcal{E}\right)=\exp \left[-\frac{2}{\hbar} \int_{z_{-}}^{z_{+}} \kappa_{z} d z\right] .
$$

In order to derive from (20) the total rate of pair production in the electric field we must multiply it with the incident particle flux density at the entrance $z_{-}$of the tunnel. The particle velocity at that point is $v_{z}=\partial \mathcal{E} / \partial p_{z}$, where the relation between $\mathcal{E}$ and $z_{-}$is given by Eq. (4):

$$
-1=\frac{\mathcal{E}-V\left(z_{-}\right)}{\sqrt{\left(c p_{\perp}\right)^{2}+m_{e}^{2} c^{4}}} .
$$

This must be multiplied with the particle density which is given by the phase space density $d^{3} p /(2 \pi \hbar)^{3}$. The incident flux density at the tunnel entrance is therefore

$$
j_{z}\left(z_{-}\right)=D_{s} \int \frac{\partial \mathcal{E}}{\partial p_{z}} \frac{d^{2} p_{\perp}}{(2 \pi \hbar)^{2}} \frac{d p_{z}}{2 \pi \hbar}=D_{s} \int \frac{d \mathcal{E}}{2 \pi \hbar} \frac{d^{2} p_{\perp}}{(2 \pi \hbar)^{2}},
$$

and the extra factor $D_{s}$ is equal to 2 for electrons with two spin orientations [32].

It is useful to change the variable of integration from $z$ to $\zeta(z)$ defined by

$$
\zeta\left(p_{\perp}, \mathcal{E} ; z\right) \equiv \frac{\mathcal{E}-V(z)}{\sqrt{\left(c p_{\perp}\right)^{2}+m_{e}^{2} c^{4}}},
$$

and to introduce the notation for the electric field $E\left(p_{\perp}, \mathcal{E} ; \zeta\right) \equiv E\left[\bar{z}\left(p_{\perp}, \mathcal{E} ; \zeta\right)\right]$, where $\bar{z}\left(p_{\perp}, \mathcal{E} ; \zeta\right)$ is the inverse function of (24), the equations in (44) reduce to

$$
\zeta_{-}\left(p_{\perp}, \mathcal{E} ; z_{-}\right)=-1, \quad \zeta_{+}\left(p_{\perp}, \mathcal{E} ; z_{+}\right)=+1
$$


In terms of the variable $\zeta$, the WKB transmission probability (21) can be rewritten as

$$
W_{\mathrm{WKB}}\left(p_{\perp}, \mathcal{E}\right)=\exp \left\{-\frac{2 m_{e}^{2} c^{3}}{e \hbar E_{0}}\left[1+\frac{\left(c p_{\perp}\right)^{2}}{m_{e}^{2} c^{4}}\right] \int_{-1}^{1} d \zeta \frac{\sqrt{1-\zeta^{2}}}{E\left(p_{\perp}, \mathcal{E} ; \zeta\right) / E_{0}}\right\} .
$$

Here we have introduced a standard field strength $E_{0}$ to make the integral in the exponent dimensionless, which we abbreviate by

$$
G\left(p_{\perp}, \mathcal{E}\right) \equiv \frac{2}{\pi} \int_{-1}^{1} d \zeta \frac{\sqrt{1-\zeta^{2}}}{E\left(p_{\perp}, \mathcal{E} ; \zeta\right) / E_{0}}
$$

The first term in the exponent of (26) is equal to $2 E_{c} / E_{0}$, where

$$
E_{c} \equiv m_{e}^{2} c^{3} / e \hbar
$$

is the critical field strength which creates a pair over two Compton wavelengths $2 \lambda_{C}=2 \hbar / m_{e} c$.

At the semiclassical level, tunneling takes place only if the potential height is larger than $2 m_{e} c^{2}$ and for energies $\mathcal{E}$ for which there are two real turning points $z_{ \pm}$. The total tunneling rate is obtained by integrating over all incoming momenta and the total area $V_{\perp}=\int d x d y$ of the incoming flux. The WKB-rate per area is

$$
\frac{\Gamma_{\mathrm{WKB}}}{V_{\perp}}=D_{s} \int \frac{d \mathcal{E}}{2 \pi \hbar} \int \frac{d^{2} p_{\perp}}{(2 \pi \hbar)^{2}} W_{\mathrm{WKB}}\left(p_{\perp}, \mathcal{E}\right) .
$$

Using the relation following from (22)

$$
d \mathcal{E}=e E\left(z_{-}\right) d z_{-},
$$

we obtain the alternative expression

$$
\frac{\Gamma_{\mathrm{WKB}}}{V_{\perp}}=D_{s} \int \frac{d z_{-}}{2 \pi \hbar} \int \frac{d^{2} p_{\perp}}{(2 \pi \hbar)^{2}} e E\left(z_{-}\right) W_{\mathrm{WKB}}\left(p_{\perp}, \mathcal{E}\left(z_{-}\right)\right)
$$

where $\mathcal{E}\left(z_{-}\right)$is obtained by solving the differential equation (30).

The integral over $p_{\perp}$ cannot be done exactly. At the semiclassical level, this is fortunately not necessary. Since $E_{c}$ is proportional to $1 / \hbar$, the exponential in (26) restricts the transverse momentum $p_{\perp}$ to be small of the order of $\sqrt{\hbar}$, so that the integral in (31) may be calculated from an expansion of $G\left(p_{\perp}, \mathcal{E}\right)$ up to the order $p_{\perp}^{2}$ :

$$
G\left(p_{\perp}, \mathcal{E}\right) \simeq \frac{2}{\pi} \int_{-1}^{1} d \zeta \frac{\sqrt{1-\zeta^{2}}}{E(0, \mathcal{E} ; \zeta) / E_{0}}\left[1-\frac{1}{2} \frac{d E(0, \mathcal{E}, \zeta) / d \zeta}{E(0, \mathcal{E}, \zeta)} \zeta \delta+\ldots\right]=G(0, \mathcal{E})+G_{\delta}(0, \mathcal{E}) \delta+\ldots
$$

where $\delta \equiv \delta\left(p_{\perp}\right) \equiv\left(c p_{\perp}\right)^{2} /\left(m_{e}^{2} c^{4}\right)$, and

$$
\begin{aligned}
G_{\delta}(0, \mathcal{E}) & \equiv-\frac{1}{\pi} \int_{-1}^{1} d \zeta \frac{\zeta \sqrt{1-\zeta^{2}}}{E^{2}(0, \mathcal{E} ; \zeta) / E_{0}} E^{\prime}(0, \mathcal{E} ; \zeta) \\
& =-\frac{1}{2} G(0, \mathcal{E})+\frac{1}{\pi} \int_{-1}^{1} d \zeta \frac{\zeta^{2}}{\sqrt{1-\zeta^{2}}} \frac{d \zeta}{E(0, \mathcal{E}, \zeta) / E_{0}}
\end{aligned}
$$


We can now perform the integral over $\mathbf{p}_{\perp}$ in (31) approximately as follows:

$$
\begin{aligned}
\int \frac{d^{2} p_{\perp}}{(2 \pi \hbar)^{2}} e^{-\pi\left(E_{c} / E_{0}\right)(1+\delta)\left[G(0, \mathcal{E})+G_{\delta}(0, \mathcal{E}) \delta\right]} & \approx \frac{m_{e}^{2} c^{2}}{4 \pi \hbar^{2}} \int_{0}^{\infty} d \delta e^{-\pi\left(E_{c} / E_{0}\right)[G(0, \mathcal{E})+\delta \tilde{G}(0, \mathcal{E})} \\
& =\frac{e E_{0}}{4 \pi^{2} \hbar c \tilde{G}(0, \mathcal{E})} e^{-\pi\left(E_{c} / E_{0}\right) G(0, \mathcal{E})}
\end{aligned}
$$

where

$$
\tilde{G}(0, \mathcal{E}) \equiv G(0, \mathcal{E})+G_{\delta}(0, \mathcal{E})
$$

The electric fields $E\left(p_{\perp}, \mathcal{E} ; \zeta\right)$ at the tunnel entrance $z_{-}$in the prefactor of (31) can be expanded similarly to first order in $\delta$. If $z_{-}^{0}$ denotes the solutions of (22) at $p_{\perp}=0$, we see that for small $\delta$ :

$$
\Delta z_{-} \equiv z_{-}-z_{-}^{0} \approx \frac{m_{e} c^{2}}{E\left(z_{-}^{0}\right)} \frac{\delta}{2}
$$

so that

$$
E\left(z_{-}\right) \simeq E\left(z_{-}^{0}\right)-m_{e} c^{2} \frac{E^{\prime}\left(z_{-}^{0}\right)}{E\left(z_{-}^{0}\right)} \frac{\delta}{2}
$$

Here the extra term proportional to $\delta$ can be neglected in the semiclassical limit since it gives a contribution to the prefactor of the order $\hbar$. Thus we obtain the WKB-rate (31) of pair production per unit area

$$
\frac{\Gamma_{\mathrm{WKB}}}{V_{\perp}} \equiv \int d z \frac{\partial_{z} \Gamma_{\mathrm{WKB}}(z)}{V_{\perp}} \simeq D_{s} \int d z \frac{e^{2} E_{0} E(z)}{8 \pi^{3} \hbar^{2} c \tilde{G}(0, \mathcal{E}(z))} e^{-\pi\left(E_{c} / E_{0}\right) G(0, \mathcal{E}(z))}
$$

where $z$ is short for $z_{-}^{0}$. At this point it is useful to return from the integral $\int d z_{-} e E\left(z_{-}\right)$introduced in (31) to the original energy integral $\int d \mathcal{E}$ in (29), so that the final result is

$$
\frac{\Gamma_{\mathrm{WKB}}}{V_{\perp}} \equiv \int d \mathcal{E} \frac{\partial_{\mathcal{E}} \Gamma_{\mathrm{WKB}}(z)}{V_{\perp}} \simeq D_{s} \frac{e E_{0}}{4 \pi^{2} \hbar c} \int \frac{d \mathcal{E}}{2 \pi \hbar} \frac{1}{\tilde{G}(0, \mathcal{E})} e^{-\pi\left(E_{c} / E_{0}\right) G(0, \mathcal{E})}
$$

where $\mathcal{E}$-integration is over all crossing energy-levels.

These formula can be approximately applied to the 3-dimensional case of electric fields $\mathbf{E}(x, y, z)$ and potentials $V(x, y, z)$ at the points $(x, y, z)$ where the tunneling length $a \equiv z_{+}-z_{-}$is much smaller than the variation lengths $\delta x_{\perp}$ of electric potentials $V(x, y, z)$ in the $x y$-plane,

$$
\frac{1}{a} \gg \frac{1}{V} \frac{\delta V}{\delta x_{\perp}}
$$

At these points $(x, y, z)$, we can arrange the tunneling path $d z$ and momentum $p_{z}(x, y, z)$ in the direction of electric field, corresponding perpendicular area $d^{2} V_{\perp} \equiv d x d y$ for incident flux and perpendicular momentum $\mathbf{p}_{\perp}$. It is then approximately reduced to a one-dimensional problem in 
the region of size $\mathcal{O}(a)$ around these points. The surfaces $z_{-}\left(x_{-}, y_{-}, \mathcal{E}\right)$ and $z_{+}=\left(x_{+}, y_{+}, \mathcal{E}\right)$ assciated with the classical turning points are determined by Eqs. (24) and Eqs. (25) for a given energy $\mathcal{E}$. The WKB-rate of pair production (38) can then be expressed as an volume integral over the rate density per volume element

$$
\Gamma_{\mathrm{WKB}}=\int d x d y d z \frac{d^{3} \Gamma_{\mathrm{WKB}}}{d x d y d z}=\int d t d x d y d z \frac{d^{4} N_{\mathrm{WKB}}}{d t d x d y d z}
$$

On the right-hand side we have found it useful to rewrite the rate $\Gamma_{\mathrm{WKB}}$ as the time derivative of the number of pair creation events $d N_{\mathrm{WKB}} / d t$, so that we obtain an event density in four-space

$$
\frac{d^{4} N_{\mathrm{WKB}}}{d t d x d y d z} \approx D_{s} \frac{e^{2} E_{0} E(z)}{8 \pi^{3} \hbar \tilde{G}(0, \mathcal{E}(z))} e^{-\pi\left(E_{c} / E_{0}\right) G(0, \mathcal{E}(z))},
$$

Here $x, y$ and $z$ are related by the function $z=z_{-}(x, y, \mathcal{E})$ which is obtained by solving (30).

It is now useful to observe that the left-hand side of (42) is a Lorentz-invariant quantity. In addition, it is symmetric under the exchange of time and $z$, and this symmetry will be exploited in the next section to relate pair production processes in a $z$-dependent electric field $E(z)$ to those in a time-dependent field $E(t)$.

Attempts to go beyond the WKB results (38) or (39) require a great amount of work. Corrections will come from three sources:

I from the higher terms of order in $(\hbar)^{n}$ with $n>1$ in the the expansion (10) solving the Riccati equation (8).

II from the perturbative evaluation of the integral over $\mathbf{p}_{\perp}$ in Eqs. (29) or (31) when going beyond the Gaussian approximation.

III from perturbative corrections to the Gaussian energy integral (39) or the corresponding $z$-integral (38).

All these corrections contribute terms of higher order in $\hbar$.

\section{Including a Smoothly Varying $\mathbf{B}(z)$-Field Parallel to $\mathbf{E}(z)$}

The above results can easily be extended to allow for the presence of a constant magnetic field $\mathbf{B}$ parallel to $\mathbf{E}(z)$. Then the wave function factorizes into a Landau state and a spinor function first calculated by Sauter [1]. In the WKB approximation, the energy spectrum is still given by 
Eq. (2), but the squared transverse momenta $p_{\perp}^{2}$ is quantized and must be replaced by discrete values corresponding to the Landau energy levels. From the known nonrelativistic levels for the Hamiltonian $p_{\perp}^{2} / 2 m_{e}$ we extract immediately the replacements

$$
c^{2} p_{\perp}^{2}=2 m_{e} c^{2} \times\left(\frac{p_{\perp}^{2}}{2 m_{e}}\right) \longrightarrow 2 m_{e} c^{2} \times\left[\hbar \omega_{L}\left(n+\frac{1}{2}+g \sigma\right)\right], n=0,1,2, \cdots,
$$

where $g=2+\alpha / \pi+\ldots$ is the anomalous magnetic moment of the electron, $\omega_{L}=e B / m_{e} c$ the Landau frequency, with $\sigma= \pm 1 / 2$ for spin- $1 / 2$ and $\sigma=0$ for spin- 0 , which are eigenvalues of the Pauli matrix $\sigma_{z}$. The quantum number $n$ characterizing the Landau levels counts the levels of the harmonic oscillations in the plane orthogonal to the $z$-direction. Apart from the replacement (43), the WKB calculations remain the same. Thus we must only replace the integration over the transverse momenta $\int d^{2} p_{\perp} /(2 \pi \hbar)^{2}$ in Eq. (34) by the sum over all Landau levels with the degeneracy $e B /(2 \pi \hbar c)$. Thus, the right-hand side becomes

$$
\frac{e B}{2 \pi \hbar c} e^{-\pi\left(E_{c} / E_{0}\right) G(0, \mathcal{E})} \sum_{n, \sigma} e^{-\pi\left(B / E_{0}\right)(n+1 / 2+g \sigma) \tilde{G}(0, \mathcal{E})} .
$$

The result is, for spin- 0 and spin- $1 / 2$ :

$$
\frac{e E_{0}}{4 \pi^{2} \hbar c \tilde{G}(0, \mathcal{E})} e^{-\pi\left(E_{c} / E_{0}\right) G(0, \mathcal{E})} f_{0,1 / 2}\left(B \tilde{G}(0, \mathcal{E}) / E_{0}\right)
$$

where

$$
f_{0}(x) \equiv \frac{\pi x}{\sinh \pi x}, \quad f_{1 / 2}(x) \equiv 2 \frac{\pi x}{\sinh \pi x} \cosh \frac{\pi g x}{2}
$$

In the limit $B \rightarrow 0$, Eq. (46) reduces to Eq. (34).

The result remains approximately valid if the magnetic field has a smooth $z$-dependence varying little over a Compton wavelength $\lambda_{e}$.

In the following we shall focus only on nonuniform electric fields without a magnetic field.

\section{TIME-DEPENDENT ELECTRIC FIELDS}

The above semiclassical considerations can be applied with little change to the different physical situation in which the electric field along the $z$-direction depends only on time rather than $z$. Instead of the time $t$ itself we shall prefer working with the zeroth length coordinate $x_{0}=c t$, as usual in relativistic calculations. As an intermediate step consider for a moment a vector potential

$$
A_{\mu}=\left(A_{0}(z), 0,0, A_{z}\left(x_{0}\right)\right)
$$


with the electric field

$$
E=-\partial_{z} A_{0}(z)-\partial_{0} A_{z}\left(x_{0}\right), \quad x_{0} \equiv c t
$$

The associated Klein-Gordon equation (5) reads

$$
\left\{\left[i \hbar \partial_{0}+\frac{e}{c} A_{0}(z)\right]^{2}+\hbar^{2} \partial_{\mathbf{x}_{\perp}}^{2}-\left[i \hbar \partial_{z}+\frac{e}{c} A_{z}\left(x_{0}\right)\right]^{2}-m_{e}^{2} c^{2}\right\} \phi(x)=0 .
$$

The previous discussion was valid under the assumption $A_{z}\left(x_{0}\right)=0$, in which case the ansatz

$\phi(x)=e^{-i \mathcal{E} t / \hbar} e^{i \mathbf{p}_{\perp} \mathbf{x}_{\perp}} \phi_{\mathbf{p}_{\perp}, \mathcal{E}}(z)$ led to the field equation (6) . For the present discussion it is useful to write the ansatz as $\phi(x)=e^{-i p_{0} x_{0} / \hbar} e^{i \mathbf{p}_{\perp} \mathbf{x}_{\perp} / \hbar} \phi_{\mathbf{p}_{\perp}, p_{0}}(z)$ with $p_{0}=\mathcal{E} / c$, and Eq. (6) in the form

$$
\left\{\frac{1}{c^{2}}\left[\mathcal{E}-e \int^{z} d z^{\prime} E\left(z^{\prime}\right)\right]^{2}-p_{\perp}^{2}-m_{e}^{2} c^{2}+\hbar^{2} \frac{d^{2}}{d z^{2}}\right\} \phi_{\mathbf{p}_{\perp}, p_{0}}(z)=0 .
$$

Now we assume the electric field to depend only on $x_{0}=c t$. Then the ansatz $\phi(x)=$ $e^{i p_{z} z / \hbar} e^{i \mathbf{p}_{\perp} \mathbf{x}_{\perp} / \hbar} \phi_{\mathbf{p}_{\perp}, p_{z}}\left(x_{0}\right)$ leads to the field equation

$$
\left\{-\hbar^{2} \frac{d^{2}}{d x_{0}^{2}}-p_{\perp}^{2}-m_{e}^{2} c^{2}-\left[-p_{z}-\frac{e}{c} \int^{x_{0}} d x_{0}^{\prime} E\left(x_{0}^{\prime}\right)\right]^{2}\right\} \phi_{\mathbf{p}_{\perp}, p_{z}}\left(x_{0}\right)=0 .
$$

If we compare Eq. (51) with (50) we realize that one arises from the other by interchanging

$$
z \leftrightarrow x_{0}, \quad p_{\perp} \rightarrow i p_{\perp}, \quad c \rightarrow i c, \quad E \rightarrow-i E
$$

With these exchanges we may easily calculate the decay rate of the vacuum caused by a timedependent electric field $E\left(x_{0}\right)$ using the above-derived formulas.

\section{APPLICATIONS}

We now apply Formulas (39) or (38) to various external field configurations capable of producing electron-positron pairs.

\section{A. Step-like electric field}

First we check our result for the original case of a constant electric field $E(z) \equiv e E_{0}$ where the potential energy is the linear function $V(z)=-e E_{0} z$. Here the function (27) becomes trivial

$$
G(0, \mathcal{E})=\frac{2}{\pi} \int_{-1}^{1} d \zeta \sqrt{1-\zeta^{2}}=1, \quad G_{\delta}(0, \mathcal{E})=0
$$


which is independent of $\mathcal{E}$ ( or $z_{-}$). The WKB-rate for pair-production per unit time and volume is found from Eq. (38) to be

$$
\frac{\Gamma_{\mathrm{WKB}}^{\mathrm{EH}}}{V} \simeq D_{s} \frac{e^{2} E_{0}^{2}}{8 \pi^{3} \hbar^{2} c} e^{-\pi E_{c} / E_{0}}
$$

where $V \equiv d z_{-} V_{\perp}$. This expression contains the exponential $e^{-\pi E_{c} / E_{0}}$ found by Sauter [1], and the correct prefactor as calculated by Heisenberg and Euler [2], and by Schwinger [3].

In order to apply the translation table (52) to obtain the analogous result for the constant electric field in time, we rewrite Eq. (54) as

$$
\frac{d N_{\mathrm{WKB}}}{d x_{0} V} \simeq D_{s} \frac{e^{2} E_{0}^{2}}{8 \pi^{3} \hbar^{2} c^{2}} e^{-\pi E_{c} / E_{0}},
$$

where $d N_{\mathrm{WKB}} / d x_{0}=\Gamma_{\mathrm{WKB}}^{\mathrm{EH}} / c$ and $N_{\mathrm{WKB}}$ is the number of pairs produced. Applying the translation table (52) to Eq. (55), one obtains the same formula as Eq. (54).

\section{B. Sauter electric field}

Let us now consider the nontrivial Sauter electric field localized within finite slab in the $x y$ plane with the width $\ell$ in the $z$-direction. A field of this type can be produced, e.g., between two opposite charged conducting plates. The electric field $E(z) \hat{\mathbf{z}}$ in the $z$-direction and the associated potential energy $V(z)$ are given by

$$
E(z)=E_{0} / \cosh ^{2}(z / \ell), \quad V(z)=-\sigma m_{e} c^{2} \tanh (z / \ell)
$$

where

$$
\sigma \equiv e E_{0} \ell / m_{e} c^{2}=\left(\ell / \lambda_{C}\right)\left(E_{0} / E_{c}\right)
$$

From now on we shall use natural units in which energies are measured in units of $m_{e} c^{2}$. Figure 1 shows the positive and negative-energy spectra $\mathcal{E}_{ \pm}(z)$ of Eq. (2) for $p_{z}=p_{\perp}=0$ to show the energy-gap and energy-level crossings. From Eq. (44) we find the classical turning points

$$
z_{ \pm}=\ell \operatorname{arctanh} \frac{\mathcal{E} \pm \sqrt{1+\delta}}{\sigma}=\frac{\ell}{2} \ln \frac{\sigma+\mathcal{E} \pm \sqrt{1+\delta}}{\sigma-\mathcal{E} \mp \sqrt{1+\delta}}
$$

Tunneling is possible for all energies satisfying

$$
-\sqrt{1+\delta}+\sigma \geq \mathcal{E} \geq \sqrt{1+\delta}-\sigma
$$

for the strength parameter $\sigma>\sqrt{1+\delta}$. 
We may invert Eq. (24) to find the relation between $\zeta$ and $z$ :

$$
z=z\left(p_{\perp}, \mathcal{E} ; \zeta\right)=\ell \operatorname{arctanh} \frac{\mathcal{E}+\zeta \sqrt{1+\delta}}{\sigma}=\frac{\ell}{2} \ln \frac{\sigma+\mathcal{E}+\zeta \sqrt{1+\delta}}{\sigma-\mathcal{E}-\zeta \sqrt{1+\delta}}
$$

In terms of the function $z\left(p_{\perp}, \mathcal{E} ; \zeta\right)$, the equation (58). reads simply $z_{ \pm}=z\left(p_{\perp}, \mathcal{E} ; \pm 1\right)$.

Inserting (60) into the equation for $E(z)$ in Eq. (56), we obtain

$$
E(z)=E_{0}\left[1-\left(\frac{\zeta \sqrt{1+\delta}-\mathcal{E}}{\sigma}\right)^{2}\right] \equiv E\left(p_{\perp}, \mathcal{E} ; \zeta\right) .
$$

We now calculate $G(0, \mathcal{E})$ and $G_{\delta}(0, \mathcal{E})$ of Eqs. (27), (32) and (33):

$$
G(0, \mathcal{E})=2 \sigma^{2}-\sigma\left[(\sigma-\mathcal{E})^{2}-1\right]^{1 / 2}-\sigma\left[(\sigma+\mathcal{E})^{2}-1\right]^{1 / 2}
$$

and

$$
G(0, \mathcal{E})+G_{\delta}(0, \mathcal{E})=\frac{\sigma}{2}\left\{\left[(\sigma-\mathcal{E})^{2}-1\right]^{-1 / 2}+\left[(\sigma+\mathcal{E})^{2}-1\right]^{-1 / 2}\right\}
$$

Substituting the functions $G(0, \mathcal{E})$ and $G_{\delta}(0, \mathcal{E})$ into Eqs. (38) and (39), we obtain the general expression for the pair-production rate per volume slice at a given tunnel entrance point $z_{-}(\mathcal{E})$ or the associated energy $\mathcal{E}\left(z_{-}\right)$. The pair-production rate per area is obtained by integrating over all slices permitted by the energy inequality (59).

In Fig. 2 we show the slice dependence of the integrand in the tunneling rate (38) for the Sauter potential (56) and compare it with the constant-field expression (54) of Euler and Heisenberg, if this is evaluated at the $z$-dependent electric field $E(z)$. This is done once as a function of the tunnel entrance point $z$ and once as a function of the associated energy $\mathcal{E}$. On each plot, the difference between the two curves illustrates the nonlocality of the tunneling process [33].

The integral is dominated by the region around $\mathcal{E} \sim 0$, where the tunneling length is shortest [see Fig. [1] and tunneling probability is largest. Both functions $G(0, \mathcal{E})$ and $G_{\delta}(0, \mathcal{E})$ have a symmetric peak at $\mathcal{E}=0$. Around the peak they can be expanded in powers of $\mathcal{E}$ as

$$
G(0, \mathcal{E})=2\left[\sigma^{2}-\sigma\left(\sigma^{2}-1\right)^{1 / 2}\right]+\frac{\sigma}{\left(\sigma^{2}-1\right)^{3 / 2}} \mathcal{E}^{2}+\mathcal{O}\left(\mathcal{E}^{4}\right)=G_{0}(\sigma)+\frac{1}{2} G_{2}(\sigma) \mathcal{E}^{2}+\mathcal{O}\left(\mathcal{E}^{4}\right),
$$

and

$$
G(0, \mathcal{E})+G_{\delta}(0, \mathcal{E})=\frac{\sigma}{\left(\sigma^{2}-1\right)^{1 / 2}}+\frac{1}{2} \frac{\left(1+2 \sigma^{2}\right)}{\left(\sigma^{2}-1\right)^{5 / 2}} \mathcal{E}^{2}+\mathcal{O}\left(\mathcal{E}^{4}\right)=\bar{G}_{0}(\sigma)+\frac{1}{2} \bar{G}_{2}(\sigma) \mathcal{E}^{2}+\mathcal{O}\left(\mathcal{E}^{4}\right)
$$

The exponential $e^{-\pi G(0, \mathcal{E}) E_{c} / E_{0}}$ has a Gaussian peak around $\mathcal{E}=0$ whose width is of the order of $1 / E_{c} \propto \hbar$. This implies that in the semiclassical limit, we may perform only a Gaussian integral 

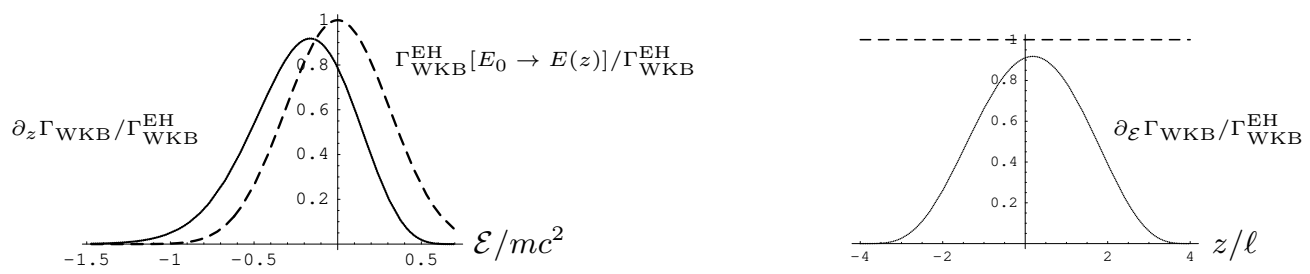

FIG. 2: We plot the slice dependence of the integrand in the tunneling rate (38) for the Sauter potential $(\underline{56})$ : left, as a function of the tunnel entrance $z$ (compare with numeric results plotted in Fig. 1 of Ref. 26]); right, as a function of the associated energy $\mathcal{E}$, which is normalized by the Euler-Heisenberg rate (54). The dashed curve in left figure shows the Euler-Heisenberg expression (54) evaluated for the $z$-dependent field $E(z)$ to illustrate the nonlocality of the production rate. The dashed curve in right figure shows the Euler-Heisenberg expression (54) is independent of energy-level crossing $\mathcal{E}$. The dimensionless parameters are $\sigma=5, E_{0} / E_{c}=1$.

and neglect the $\mathcal{E}$-dependence of the prefactor in (39). Recalling that $\mathcal{E}$ in this section is in natural units with $m_{e} c^{2}=1$, we must replace $\int d \mathcal{E}$ by $m_{e} c^{2} \int d \mathcal{E}$ and can perform the integral over $\mathcal{E}$ approximately as follows

$$
\frac{\Gamma_{\mathrm{WKB}}}{V_{\perp}} \simeq D_{s} \frac{e E_{0} m_{e} c^{2}}{4 \pi^{2} \hbar c} \frac{1}{\bar{G}_{0}} e^{-\pi\left(E_{c} / E_{0}\right) G_{0}} \int \frac{d \mathcal{E}}{2 \pi \hbar} e^{-\pi\left(E_{c} / E_{0}\right) G_{0}^{\prime \prime} \mathcal{E}^{2} / 2}=D_{s} \frac{e E_{0}}{4 \pi^{2} \hbar c} \frac{1}{\bar{G}_{0}} \frac{e^{-\pi\left(E_{c} / E_{0}\right) G_{0}}}{2 \pi \hbar \sqrt{G_{0}^{\prime \prime} E_{c} / 2 E_{0}}} .
$$

For convenience, we have extended the limits of integration over $E$ from the interval $(-1+\sigma, 1-\sigma)$ to $(-\infty, \infty)$. This introduces exponentially small errors and can be ignored.

Using the relation (57) we may replace $e E_{0} m_{e} c^{2} / \hbar c$ by $e^{2} E_{0}^{2} 2 \ell / \sigma$, and obtain

$$
\frac{\Gamma_{\mathrm{WKB}}[\text { total }]}{V_{\perp} \ell} \simeq D_{s} \frac{e^{2} E_{0}^{2}}{8 \pi^{3} \hbar^{2} c} \sqrt{\frac{E_{0}}{E_{c}}} \frac{\left(\sigma^{2}-1\right)^{5 / 4}}{\sigma^{5 / 2}} e^{-\pi G_{0}(\sigma) E_{c} / E_{0}}
$$

This approximate result agrees [34] with that obtained before with a different, somewhat more complicated technique proposed by Dunne and Schubert [23] after the fluctuation determinant was calculated exactly in [24] with the help of the Gelfand-Yaglom method following Ref. [35]. The advantage of knowing the exact fluctuation determinant could not, however, be fully exploited since the remaining integral was calculated only in the saddle point approximation. The rate (67) agrees with the leading term of the expansion (42) of Kim and Page [21]. Note that the higher expansion terms calculated by the latter authors do not yet lead to proper higher-order results since they are only of type II and III in the list after Eq. (42). The terms of equal order in $\hbar$ in the expansion (10) of the solution of the Riccatti equation are still missing.

Using the translation table (52), it is straightforward to obtain the pair-production rate of the Sauter-type of electric field depending on time rather than space. According to the translation 
table (52), we have to replace $\ell \rightarrow c \mathcal{T}$, where $\mathcal{T}$ is the characteristic time over which the electric field acts - the analog of $\ell$ in (56). Thus the field (56) becomes

$$
E(t)=E_{0} / \cosh ^{2}(t / \mathcal{T}), \quad V(t)=-\tilde{\sigma} m_{e} c^{2} \tanh (t / \mathcal{T})
$$

According to the same table we must also replace $\sigma \rightarrow i \tilde{\sigma}$, where

$$
\tilde{\sigma} \equiv e E_{0} \mathcal{T} / m_{e} c
$$

This brings $G_{0}(\sigma)$ of Eq. (62) to the form

$$
G_{0}(\sigma) \rightarrow G_{0}^{t}(\tilde{\sigma})=2\left[\tilde{\sigma}\left(\tilde{\sigma}^{2}-1\right)^{1 / 2}-\tilde{\sigma}^{2}\right]
$$

and yields the pair-production rate

$$
\frac{\Gamma_{\mathrm{WKB}}^{z}[\text { total }]}{V_{\perp} \mathcal{T}} \simeq D_{s} \frac{e^{2} E_{0}^{2}}{8 \pi^{3} \hbar^{2} c} \sqrt{\frac{E_{0}}{E_{c}}}\left(\frac{\tilde{\sigma}^{2}+1}{\tilde{\sigma}^{2}}\right)^{5 / 4} e^{-\pi G_{0}^{t}(\tilde{\sigma}) E_{c} / E_{0}},
$$

where $\Gamma_{\mathrm{WKB}}^{z}[$ total $]=\partial \mathrm{N}_{\mathrm{WKB}} / \partial \mathrm{z}$ is the number of pairs produced per unit thickness in a spatial shell parallel to the $x y$-plane. This agrees with Ref. [24].

Note also that the constant-field result (54) of Euler and Heisenberg cannot be deduced from (67) by simply taking the limit $\ell \rightarrow \infty$ as one might have expected. The reason is that the saddle point approximation (66) to the integral (39) becomes invalid in this limit. Indeed, if $\ell \propto \sigma$ is large in Eqs. (62) and (63), these become

$$
G(0, \mathcal{E}) \rightarrow G(0, \mathcal{E})+G_{\delta}(0, \mathcal{E}) \rightarrow \frac{1}{1-\mathcal{E}^{2} / \sigma^{2}}
$$

and the integral in (39) becomes approximately

$$
e^{-\pi\left(E_{c} / E_{0}\right)} \int_{-\sigma}^{\sigma} \frac{d \mathcal{E}}{2 \pi \hbar}\left(1-\mathcal{E}^{2} / \sigma^{2}\right) e^{-\pi\left(E_{c} / E_{0}\right)\left(\mathcal{E}^{2} / \sigma^{2}\right)}
$$

For not too large $\ell \propto \sigma$, the integral can be evaluated in the leading Gaussian approximation

$$
\int_{-\infty}^{\infty} \frac{d \mathcal{E}}{2 \pi \hbar} e^{-\pi\left(E_{c} / E_{0}\right)\left(\mathcal{E}^{2} / \sigma^{2}\right)}=\frac{1}{2 \pi \hbar} \sqrt{\frac{E_{0}}{E_{c}}} \sigma
$$

corresponding to the previous result (67) for large- $\sigma$. For a constant field, however, where the integrands becomes flat, the Gaussian approximation is no longer applicable. Instead we must first set $\sigma \rightarrow \infty$ in the integrand of (73), making it constant. Then the integral (73) becomes [36]

$$
e^{-\pi\left(E_{c} / E_{0}\right)} 2 \sigma / 2 \pi \hbar=e^{-\pi\left(E_{c} / E_{0}\right)} 2 \ell e E_{0} / m_{e} c^{2} 2 \pi \hbar .
$$


Inserting this into (39) we recover the constant-field result (54). We must replace $2 \ell$ by $L$ to comply with the relation (30) from which we obtain

$$
\int d \mathcal{E}=\int d z e E(z)=e E_{0} \int d z / \cosh ^{2}(z / \ell)=2 \ell e E_{0}=L e E_{0} .
$$

In order to see the boundary effect on the pair-production rate, we close this section with a comparison between pair-production rates in the constant field (54) and Sauter field (67) for the same field strength $E_{0}$ in the volume $V_{\perp} \ell$. The ratio $R_{\text {rate }}$ of pair-production rates (67) and (54) in the volume $V_{\perp} \ell$ is defined as

$$
R_{\text {rate }}=\sqrt{\frac{E_{0}}{E_{c}}} e^{\pi E_{c} / E_{0}} \frac{\left(\sigma^{2}-1\right)^{5 / 4}}{\sigma^{5 / 2}} e^{-\pi G_{0}(\sigma) E_{c} / E_{0}} .
$$

The soft boundary of the Sauter field (56) reduces its pair-production rate with respect to the pair-production rate (54) computed in a constant field of width $L=2 \ell$. The reduction is shown quantitatively in Fig. 3, where curves are plotted for the rates (54) and (67), and and for their ratio (76) at $E_{0}=E_{c}$ and $\sigma=\ell / \lambda_{C}$ [recall (57)]. We see that the reduction is significant if the width of the field slab shrinks to the size of a Compton wavelength $\lambda_{C}$.
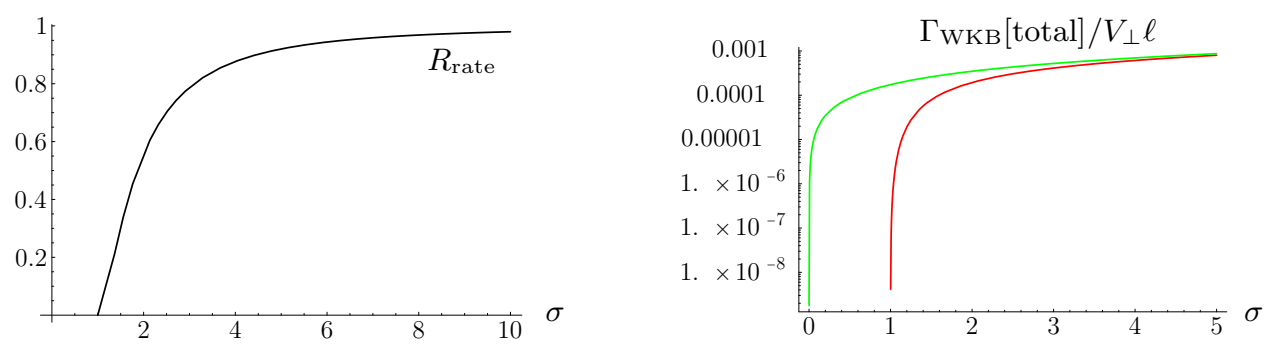

FIG. 3: Left: Ratio $R_{\text {rate }}$ defined in Eq. (76) is plotted as function of $\sigma$ in the left figure. Right: Number of pairs created in slab of Compton width per area and time as functions of $\sigma$. Upper curve is for the constant field (54), lower for the Sauter field (67)). Both plots are for $E_{0}=E_{c}$ and $\sigma=\ell / \lambda_{C}$.

\section{Constant electric field for $z>0$}

As a second application consider an electric field which is zero for $z<0$ and goes to $-E_{0}$ over a distance $\ell$ as follows:

$$
E(z)=-\frac{E_{0}}{2}\left[\tanh \left(\frac{z}{\ell}\right)+1\right], \quad V(z)=-\frac{\sigma}{2} m_{e} c^{2}\left\{\ln \cosh \left(\frac{z}{\ell}\right)+\frac{z}{\ell}\right\}
$$




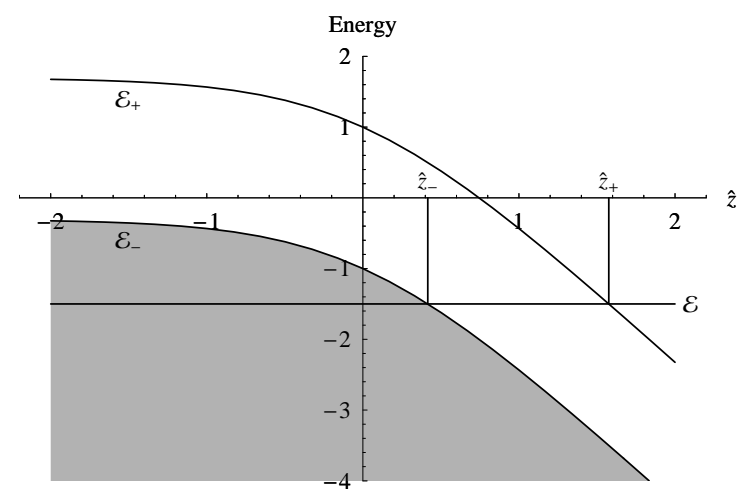

FIG. 4: Energies (2) for a soft electric field step $E(z)$ of Eq. (77) and the potentials $V_{ \pm}(z)$ (77) for $\sigma=5$. Positive and negative-energies $\mathcal{E}_{ \pm}(z)$ of Eq. (2) are plotted for $p_{z}=p_{\perp}=0$ as functions of $\hat{z}=z / \ell$.

where $\sigma \equiv e E_{0} \ell / m_{e} c^{2}$. In Fig. 4, we have plotted the positive and negative-energy spectra $\mathcal{E}_{ \pm}(z)$ defined by Eq. (2) for $p_{z}=p_{\perp}=0$ to show energy gap and level crossing. From Eq. (4) we find now the classical turning points [instead of (58)]

$$
z_{ \pm}=\frac{\ell}{2} \ln \left[2 e^{(\mathcal{E} \pm \sqrt{1+\delta}) / \sigma}-1\right]
$$

For tunneling to take place, the energy $\mathcal{E}$ has to satisfy

$$
\mathcal{E} \leq \sqrt{1+\delta}-\sigma \ln 2
$$

and $\sigma$ must be larger than $\sqrt{1+\delta} \zeta$. Expressing $z / \ell$ in terms of $\zeta$ as

$$
z=z\left(p_{\perp}, \mathcal{E} ; \zeta\right)=\frac{\ell}{2} \ln \left[2 e^{(\mathcal{E}+\zeta \sqrt{1+\delta}) / \sigma}-1\right]
$$

so that $z_{ \pm}=z\left(p_{\perp}, \mathcal{E} ; \pm 1\right)$, we find the electric field in the form

$$
E(z)=E_{0}\left[1-\frac{1}{2} e^{(\zeta \sqrt{1+\delta}-\mathcal{E}) / \sigma}\right] \equiv E\left(p_{\perp}, \mathcal{E} ; \zeta\right)
$$

Inserting this into Eq. (27) and expanding $E_{0} / E\left(p_{\perp}, \mathcal{E} ; \zeta\right)$ in powers we obtain

$$
G\left(p_{\perp}, \mathcal{E}\right)=1+\sum_{n=1}^{\infty} \frac{e^{-n \mathcal{E} / \sigma}}{2^{n}} \frac{2}{\pi} \int_{-1}^{1} d \zeta \sqrt{1-\zeta^{2}} e^{n \hat{\zeta} / \sigma}=1+\sum_{n=1}^{\infty} e^{-n \mathcal{E} / \sigma} I_{1}(n \sqrt{1+\delta} / \sigma),
$$

where $I_{1}(x)$ is a modified Bessel function. Expanding $I_{1}(n \sqrt{1+\delta} / \sigma)$ in powers of $\delta$ :

$$
I_{1}(n \sqrt{1+\delta} / \sigma)=I_{1}(n / \sigma)+(n / 4 \sigma)\left[I_{0}(n / \sigma)+I_{2}(n / \sigma)\right] \delta+\ldots
$$


we identify

$$
\begin{aligned}
G(0, \mathcal{E}) & =1+\sum_{n=1}^{\infty} e^{-n \mathcal{E} / \sigma} I_{1}(n / \sigma) \\
G(0, \mathcal{E})+G_{\delta}(0, \mathcal{E}) & =1+\frac{1}{2} \sum_{n=1}^{\infty} e^{-n \mathcal{E} / \sigma}\left[(n / \sigma) I_{0}(n / \sigma)-I_{1}(n / \sigma)\right] .
\end{aligned}
$$

The integral over $\mathcal{E}$ in Eq. (39) starts at $\mathcal{E}_{<}=1-\sigma \log 2$ where the integrand rises from 0 to 1 as $\mathcal{E}$ exceeds a few units of $\sigma$. The derivative of $e^{-\pi\left(E_{c} / E_{0}\right) G(0, \mathcal{E})}$ drops from 1 to $e^{-\pi\left(E_{c} / E_{0}\right)}$ over this interval. Hence the derivative $\partial_{\mathcal{E}} e^{-\pi\left(E_{c} / E_{0}\right) G(0, \mathcal{E})}$ is peaked around some value $\overline{\mathcal{E}}$. Thus we perform the integral $\int d \mathcal{E} e^{-\pi\left(E_{c} / E_{0}\right) G(0, \mathcal{E})}$ by parts as

$$
\int d \mathcal{E} e^{-\pi\left(E_{c} / E_{0}\right) G(0, \mathcal{E})}=\left.\mathcal{E} e^{-\pi\left(E_{c} / E_{0}\right) G(0, \mathcal{E})}\right|_{\mathcal{E}_{<}} ^{\infty}-\int d \mathcal{E} \mathcal{E} \partial_{\mathcal{E}} e^{-\pi\left(E_{c} / E_{0}\right) G(0, \mathcal{E})}
$$

The first term can be rewritten with the help of $d \mathcal{E}=e E_{0} d z$ as $e^{-\pi\left(E_{c} / E_{0}\right)}\left|e E_{0}\right| \ell / 2$, thus giving rise to the decay rate (54) in the volume $V_{\perp} \ell / 2$, and the second term gives only a small correction to this. The second term in Eq. (86) shows that the boundary effects reduce the pair-production rate compared with the pair-production rate (54) in the constant field without any boundary.

\section{TUNNELING INTO BOUND STATES}

We turn now to the case in which instead of an outgoing wave as given (16) there is a bound state. We consider a linearly rising electric field whose potential is harmonic:

$$
E(z)=E_{0}\left(\frac{z}{\lambda_{C}}\right), \quad V(z)=\frac{e E_{0} \lambda_{C}}{2}\left(\frac{z}{\lambda_{C}}\right)^{2} .
$$

It will be convenient to parametrize the field strength $E_{0}$ in terms of a dimensionless reduced electric field $\epsilon$ as $E_{0}=\epsilon \hbar c / e \lambda_{C}^{2}=\epsilon E_{c}$. In Fig. [5 we have plotted the positive and negative-energy spectra $\mathcal{E}_{ \pm}(z)$ defined by Eq. (2) for $p_{z}=p_{\perp}=0$ to show energy gap and level crossing for $\epsilon>0$ (left) and $\epsilon<0$ (right). If $\epsilon$ is positive, Eq. (4) yields for $z>0$,

$$
z_{ \pm}=\lambda_{C} \sqrt{\frac{2}{\epsilon}}(\mathcal{E} \mp \sqrt{1+\delta})^{1 / 2}, \quad z_{+}<z_{-},
$$

and mirror-reflected turning points for $z<0$, obtained by exchanging $z_{ \pm} \rightarrow-z_{ \pm}$in (88). Negativeenergy electrons tunnel into the potential well $-z_{+}<z<+z_{+}$, where $\mathcal{E} \geq \mathcal{E}_{+}$, forming bound states. The associated positrons run off to infinity. 

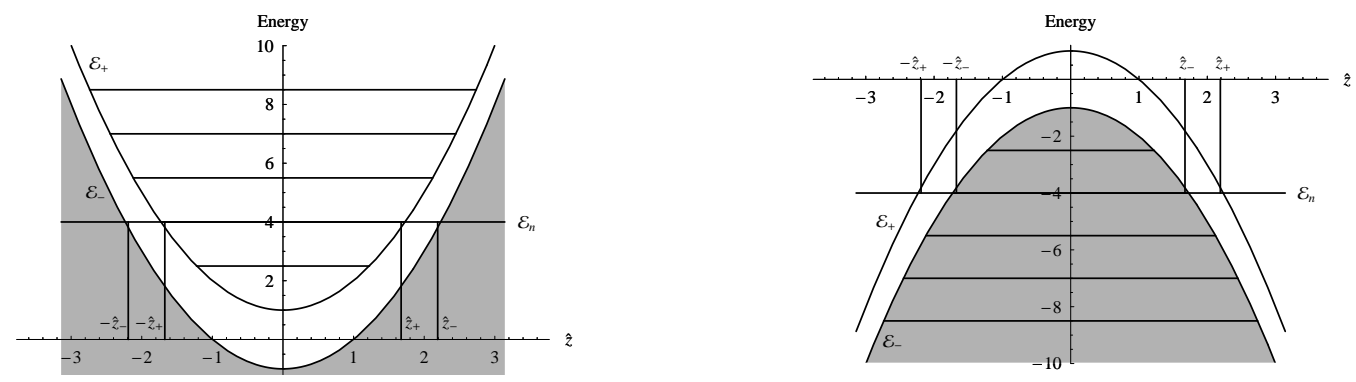

FIG. 5: Positive- and negative-energy spectra $\mathcal{E}_{ \pm}(z)$ of Eq. (2) for $p_{z}=p_{\perp}=0$ as a function of $\hat{z} \equiv z / \lambda_{C}$ for the linearly rising electric field $E(z)$ with the harmonic potential (87). The reduced field strengths are $\epsilon=2$ (left figure) and $\epsilon=-2$ (right figure). On the left, bound states are filled and positrons escape to $z= \pm \infty$. On the right, bound electrons with negative energy tunnel out of the well and escape with increasing energy to $z= \pm \infty$.

\section{A. WKB transmission probability}

Due to the physical application to be discussed in the next section, we shall study here only the tunneling process for $\epsilon>0$ on the left-hand side of Fig. 5. We consider the regime $z<0$ with the turning pints $-z_{-}<-z_{+}$. The incident wave and flux for $z<-z_{-}$pointing in the positive $z$-direction are given by Eqs. (15) and (18). The wave function for $-z_{-}<z<-z_{+}$has the form Eq. (14) with the replacement $z_{-} \rightarrow-z_{-}$. The transmitted wave is now no longer freely propagating as in (16), but a describes a bound state of a positive-energy electron:

$$
\phi_{\mathcal{E}_{n}}(z)=\frac{\mathcal{B}}{\left(p_{z}\right)^{1 / 2}} \cos \left[\frac{1}{\hbar} \int_{-z_{+}}^{z} p_{z} d z-\frac{\pi}{4}\right] .
$$

The Sommerfeld quantization condition

$$
\frac{1}{\hbar} \int_{-z_{+}}^{+z_{+}} p_{z} d z=\pi\left(n+\frac{1}{2}\right), \quad n=0,1,2, \ldots
$$

fixes the energies $\mathcal{E}_{n}$. The connection rules for the wave functions (14) and (89) at the turning point $-z_{+}$determine

$$
\mathcal{B}=\sqrt{2} \mathcal{C}_{+} e^{-i \pi n} \exp \left[-\frac{1}{\hbar} \int_{-z_{-}}^{-z_{+}} \kappa_{z} d z\right]
$$


Assuming the states $\phi_{\mathcal{E}_{n}}(z)$ to be initially unoccupied, the transmitted flux to these states at the classical turning point $-z_{+}$is

$$
\left.\frac{\hbar}{m_{e}} \phi_{\mathcal{E}_{n}}(z) \partial_{z} \phi_{\mathcal{E}_{n}}^{*}(z)\right|_{z \rightarrow-z_{+}}=\frac{|\mathcal{B}|^{2}}{\left(2 m_{e}\right)}=\frac{\left|\mathcal{C}_{+}\right|^{2}}{m_{e}} \exp \left[-\frac{2}{\hbar} \int_{-z_{-}}^{-z_{+}} \kappa_{z} d z\right] .
$$

From Eqs. (18), (92), and (20) we then find the WKB transmission probability for positrons to fill these bound states leaving a positron outside:

$$
W_{\mathrm{WKB}}\left(p_{\perp}, \mathcal{E}_{n}\right)=\exp \left[-\frac{2}{\hbar} \int_{-z_{-}}^{-z_{+}} \kappa_{z} d z\right]
$$

which has the same form as Eq. (21). The same result is obtained once more for $z>0$ with turning points $z_{+}<z_{-}$, which can be obtained from (93) by the mirror reflection $-z_{ \pm} \leftrightarrow z_{ \pm}$.

\section{B. Energy spectrum of bound states}

From Eq. (9) for $p_{z}$ and Eq. (87) for the potential $V(z)$, we calculate the eikonal (90) to determine the energy spectrum $\mathcal{E}_{n}$ of bound states

$$
\begin{aligned}
\frac{1}{\hbar} \int_{-z_{+}}^{+z_{+}} p_{z} d z & =2 \frac{\epsilon}{\lambda_{C}^{3}} \int_{0}^{z_{+}}\left[\left(z^{2}-z_{+}^{2}\right)\left(z^{2}-z_{-}^{2}\right)\right]^{1 / 2} d z \\
& =\frac{2 \epsilon z_{+}}{3 \lambda_{C}^{3}}\left[\left(z_{+}^{2}+z_{-}^{2}\right) E(t)-\left(z_{-}^{2}-z_{+}^{2}\right) K(t)\right]
\end{aligned}
$$

where $E(t), K(t)$ are complete elliptical integrals of the first and second kind, respectively, and $t \equiv z_{+} / z_{-}$. The Sommerfeld quantization rule (90) becomes

$$
\frac{8}{3}\left[\frac{2\left(\mathcal{E}_{n}-\sqrt{1+\delta}\right)}{\epsilon}\right]^{1 / 2}\left[\mathcal{E}_{n} E\left(t_{n}\right)-(\sqrt{1+\delta}) K\left(t_{n}\right)\right]=\pi\left(n+\frac{1}{2}\right) ; \quad t_{n} \equiv\left(\frac{\mathcal{E}_{n}-\sqrt{1+\delta}}{\mathcal{E}_{n}+\sqrt{1+\delta}}\right)^{1 / 2} .
$$

For any given transverse momentum $p_{\perp}=\sqrt{\delta}$, this determines the discrete energies $\mathcal{E}_{n}$.

\section{Rate of pair production}

By analogy with Eqs. (29) and (39), the transmission probability (93) must now be integrated over all incident particles with the flux (23) to yield the rate of pair production:

$$
\begin{aligned}
\frac{\Gamma_{\mathrm{WKB}}}{V_{\perp}} & =2 D_{s} \sum_{n} \frac{\omega_{n}}{2 \pi} \int \frac{d^{2} p_{\perp}}{(2 \pi \hbar)^{2}} W_{\mathrm{WKB}}\left(p_{\perp}, \mathcal{E}_{n}\right) \\
& \approx 2 D_{s} \frac{\left|e E_{0}\right|}{4 \pi^{2} \hbar c} \sum_{n} \frac{\omega_{n}}{2 \pi} \frac{1}{G\left(0, \mathcal{E}_{n}\right)+G_{\delta}\left(0, \mathcal{E}_{n}\right)} e^{-\pi\left(E_{c} / E_{0}\right) G\left(0, \mathcal{E}_{n}\right)}
\end{aligned}
$$

In obtaining these expressions we have used the energy conservation law to perform the integral over $\mathcal{E}$. This receives only contributions for $\mathcal{E}=\mathcal{E}_{n}$ where $\int d \mathcal{E}=\omega_{n} \hbar \equiv \mathcal{E}_{n}-\mathcal{E}_{n-1}$. The factor 2 
accounts for the equal contributions from the two regimes $z>0$ and $z<0$. The previous relation (30) is now replaced by

$$
\omega_{n} \hbar=\left|e E\left(z_{-}^{n}\right)\right| \Delta z_{-}^{n} .
$$

Using Eq. (24) and expressing $z / \lambda_{C}>0$ in terms of $\zeta$ as

$$
z=z\left(p_{\perp}, \mathcal{E}_{n} ; \zeta\right)=\lambda_{C} \sqrt{\frac{2}{\epsilon}}\left(\mathcal{E}_{n}-\zeta \sqrt{1+\delta}\right)^{1 / 2}
$$

we calculate $z_{ \pm}=z\left(p_{\perp}, \mathcal{E}_{n} ; \pm 1\right)$, and find the electric field in the form

$$
E(z)=E_{0} \sqrt{\frac{2}{\epsilon}}\left(\mathcal{E}_{n}-\zeta \sqrt{1+\delta}\right)^{1 / 2} \equiv E\left(p_{\perp}, \mathcal{E}_{n} ; \zeta\right)
$$

Inserting this into Eq. (27) we obtain

$$
\begin{aligned}
G\left(p_{\perp}, \mathcal{E}_{n}\right) & =\frac{2}{\pi} \sqrt{\frac{\epsilon}{2}} \int_{-1}^{1} d \zeta \frac{\sqrt{1-\zeta^{2}}}{\left[\mathcal{E}_{n}-\zeta \sqrt{1+\delta}\right]^{1 / 2}} \\
& =\frac{8}{3 \pi} \sqrt{\frac{\epsilon}{2}} \frac{\left(\mathcal{E}_{n}^{\delta}+1\right)^{1 / 2}}{(1+\delta)^{1 / 4}}\left[\left(1-\mathcal{E}_{n}^{\delta}\right) K\left(q_{n}^{\delta}\right)+\mathcal{E}_{n}^{\delta} E\left(q_{n}^{\delta}\right)\right]
\end{aligned}
$$

where $\mathcal{E}_{n}^{\delta} \equiv \mathcal{E}_{n} /(1+\delta)^{1 / 2}$ and $q_{n}^{\delta}=\sqrt{2 /\left(\mathcal{E}_{n}^{\delta}+1\right)}$. Expanding $G\left(p_{\perp}, \mathcal{E}_{n}\right)$ in powers of $\delta$ we find the zeroth order term

$$
G\left(0, \mathcal{E}_{n}\right)=\frac{8}{3 \pi} \sqrt{\frac{\epsilon}{2}}\left(\mathcal{E}_{n}+1\right)^{1 / 2}\left[\left(1-\mathcal{E}_{n}\right) K\left(q_{n}\right)+\mathcal{E}_{n} E\left(q_{n}\right)\right]
$$

and the derivative

$$
G_{\delta}\left(0, \mathcal{E}_{n}\right)=\frac{\sqrt{\epsilon}}{3 \pi} \frac{q_{n}}{\mathcal{E}_{n}} q_{n}-1\left[\left(4-5 q_{n}+\mathcal{E}_{n}\left(7-6 q_{n}\right)\right) E\left(q_{n}\right)+\left(1-\mathcal{E}_{n}-7 \mathcal{E}_{n}^{2}\right)\left(q_{n}-1\right) K\left(q_{n}\right)\right]
$$

where $q_{n} \equiv \sqrt{2 /\left(\mathcal{E}_{n}+1\right)}$.

\section{COULOMB ELECTRIC FIELD}

We now come to the physically interesting system of a bare nucleus of high charge $Z$ in the vacuum which fills its empty bound states by quantum tunneling of electrons from the negativeenergy continuum around it. The radial Coulomb field and the potential are given by

$$
e E(r)=\frac{\hat{\alpha} \hbar c}{r^{2}}, \quad V(r)=-\frac{\hat{\alpha} \hbar c}{r}
$$

where $\hat{\alpha}=Z \alpha$. The Schrödinger equation of this problem reads

$$
\left[\left(\mathcal{E}+\frac{\hat{\alpha} \hbar c}{r}\right)^{2}+c^{2} \hbar^{2} \nabla^{2}-m_{e}^{2} c^{4}\right] \psi_{\mathcal{E}}(\mathbf{x})=0 .
$$


After factorizing out spherical harmonics $\psi_{\mathcal{E}}(\mathbf{x})=R_{\mathcal{E}, l}(r) Y_{l m}(\hat{\mathbf{x}})$, the radial wave functions satisfy

$$
\left\{\mathcal{E}^{2}+c \hbar \frac{2 \mathcal{E} \hat{\alpha}}{r}+c^{2} \hbar^{2}\left[\partial_{r}^{2}-\frac{l(l+1)-\hat{\alpha}^{2}}{r^{2}}\right]-m_{e}^{2} c^{4}\right\} r R_{\mathcal{E}, l}(r)=0,
$$

where $l=0,1,2,3, \ldots$ are the quantum numbers of angular momentum. The term in brackets cannot be treated semiclassically since it contains a factor $\hbar^{2}$. This destroys the possibility of a systematic expansion of the wave function in powers of $\hbar$. In fact, the $\hbar^{2} / r^{2}$-potential should be considered as being part of the kinetic term, not of the potential. In fact, both have the same scaling dimension. There exists a heuristic way of accounting for this due to Langer [37]. He introduced a change of coordinates to $\xi \equiv \log r$, so that $\partial_{r}^{2} r R_{\mathcal{E}, l}(r)=e^{-2 \xi}\left(\partial_{\xi}^{2}-\partial_{\xi}\right) r(\xi) R_{\mathcal{E}, l}(r(\xi))=$ $e^{-2 \xi}\left(\partial_{\xi}^{2}-\frac{1}{4}\right) e^{-\xi / 2} r(\xi) R_{\mathcal{E}, l}(r(\xi))$, which brings (106) to the form

$$
\left[r^{2}(\xi)\left(\mathcal{E}^{2}-m_{e}^{2} c^{4}\right)+2 c \hbar \mathcal{E} \hat{\alpha} r(\xi)+c^{2} \hbar^{2}\left\{\partial_{\xi}^{2}-\left[l(l+1)+\frac{1}{4}-\hat{\alpha}^{2}\right]\right\}\right] \sqrt{r(\xi)} R_{\mathcal{E}, l}(r(\xi))=0,
$$

Now the $\hbar^{2} / r^{2}$-part of the kinetic term has become a trivial constant which no longer influences the semiclassical treatment [38].

Equivalently, we can apply a corrected semiclassical treatment directly to Eq. (106) if we add $\frac{1}{4}$ to $l(l+1)$ in the numerator of the centrifugal barrier. This so-called Langer correction is implemented in (106) by replacing $l(l+1)-\hat{\alpha}^{2}$ by $\lambda_{l}\left(\lambda_{l}+1\right)$, where

$$
\lambda_{l}=\left[\left(l+\frac{1}{2}\right)^{2}-\hat{\alpha}^{2}\right]^{1 / 2}-\frac{1}{2}=l-\frac{\hat{\alpha}^{2}}{2 l+1}+\mathcal{O}\left(\alpha^{4}\right) .
$$

Note that Eq. (107) has lost the singularity of Eq. (106) at the origin, a fact which is often considered to be the motivation for going to the variable $\xi$. However, due to the equal scale of $\hbar^{2} / r^{2}$ and the gradient term $\hbar^{2} \partial_{r}^{2}$, this is not the relevant property. The $\hbar^{2} / 4 r^{2}$-correction is needed at any distance to obtain the correct wave function at the semiclassical level. In fact, this wave function, although being approximate, turns out to produces the exact energy levels of the relativistic Coulomb system.

\section{Semiclassical quantization for point-like nucleus}

The semiclassical treatment of Eq. (106) with $l$ replaced by $\lambda_{l}$ starts out with the Langercorrected energy

$$
\mathcal{E}_{ \pm}\left(p_{r}, l ; r\right)= \pm \sqrt{\left(c p_{r}\right)^{2}+(\hbar c)^{2}\left(l+\frac{1}{2}\right)^{2} / r^{2}+m_{e}^{2} c^{4}}+V(r)
$$

We now impose the Sommerfeld quantization rule upon the eikonal:

$$
S(\mathcal{E})=\int_{r_{\mathrm{i}}}^{r_{\mathrm{o}}} d r p_{r}=\pi \hbar\left(n_{r}+\frac{1}{2}\right)
$$


where $n_{r}=0,1,2, \ldots$ is the radial quantum number, and

$$
p_{r}=\frac{1}{c} \sqrt{\mathcal{E}^{2}+2 c \hbar \hat{\alpha} \mathcal{E} / r-c^{2} \hbar^{2}\left(\lambda_{l}+\frac{1}{2}\right)^{2} / r^{2}-m_{e}^{2} c^{4}},
$$

whose zeros yield the turning points for each $l$ :

$$
r_{\mathrm{o}, \mathrm{i}}=\frac{c \hbar}{m_{e}^{2} c^{4}-\mathcal{E}^{2}}\left[\hat{\alpha} \mathcal{E} \pm \sqrt{\hat{\alpha}^{2} \mathcal{E}^{2}+\left(\lambda_{l}+\frac{1}{2}\right)^{2}\left(\mathcal{E}^{2}-m_{e}^{2} c^{4}\right)}\right] .
$$

Consider first the energy regime $0<\mathcal{E}<m_{e} c^{2}$. For $0<r_{\mathrm{i}}<r_{\mathrm{o}}$, we rewrite $p_{r}$ as

$$
p_{r}=\frac{1}{c} \sqrt{m_{e}^{2} c^{4}-\mathcal{E}^{2}} \frac{1}{r} \sqrt{\left(r_{\mathrm{o}}-r\right)\left(r-r_{\mathrm{i}}\right)},
$$

and perform the integral in (110) to find the eikonal

$$
S(\mathcal{E})=\frac{1}{c} \sqrt{m_{e}^{2} c^{4}-\mathcal{E}^{2}} \frac{\pi}{2}\left(r_{\mathrm{o}}+r_{\mathrm{i}}-2 \sqrt{r_{\mathrm{o}} r_{\mathrm{i}}}\right)=\pi \hbar\left[\frac{\hat{\alpha} \mathcal{E}}{\sqrt{m_{e}^{2} c^{4}-\mathcal{E}^{2}}}-\left(\lambda_{l}+\frac{1}{2}\right)\right],
$$

where

$$
r_{\mathrm{o}} r_{\mathrm{i}}=c^{2} \hbar^{2} \frac{\left(\lambda_{l}+\frac{1}{2}\right)^{2}}{m_{e}^{2} c^{4}-\mathcal{E}^{2}}, \quad r_{\mathrm{o}}+r_{\mathrm{i}}=c \hbar \frac{2 \hat{\alpha} \mathcal{E}}{m_{e}^{2} c^{4}-\mathcal{E}^{2}} .
$$

Inserting $S(\mathcal{E})$ into the quantization condition (110), we obtain the following equation for the exact bound state energies

$$
\frac{\mathcal{E}_{n l}^{2}-m_{e}^{2} c^{4}}{2 m_{e}^{2} c^{4}}=-\frac{\hat{\alpha}^{2}}{2} \frac{\mathcal{E}_{n l}{ }^{2}}{m_{e}^{2} c^{4}} \frac{1}{\left(n_{r}+\lambda_{l}+1\right)^{2}} .
$$

This is solved by

$$
\begin{aligned}
\mathcal{E}_{n l} & = \pm \frac{m_{e} c^{2}}{\sqrt{1+(Z \alpha)^{2} /\left(n-\delta_{l}\right)^{2}}} \\
& = \pm m_{e} c^{2}\left[1-\frac{\hat{\alpha}^{2}}{2 n^{2}}+\frac{3}{8} \frac{\hat{\alpha}^{4}}{n^{4}}-\frac{\hat{\alpha}^{4}}{n^{3}(2 l+1)}+\mathcal{O}\left(\hat{\alpha}^{6}\right)\right],
\end{aligned}
$$

where $n \equiv n_{r}+l+1$ is the principal quantum number of the atom, and

$$
\delta_{l}=\left(l+\frac{1}{2}\right)-\left(\lambda_{l}+\frac{1}{2}\right)=\frac{\hat{\alpha}^{2}}{2 l+1}+\mathcal{O}\left(\hat{\alpha}^{4}\right) .
$$

Although derived by a semiclassical approximation, these happen to be the exact values, due to the Langer correction in (111).

For a Dirac particle, the energy-spectrum $\mathcal{E}_{n j}$ can be obtained from a slight modification of Eq. (117):

$$
\mathcal{E}_{n j}= \pm \frac{m_{e} c^{2}}{\sqrt{1+(Z \alpha)^{2} /\left(n-\delta_{j}\right)^{2}}}
$$

where $j=\frac{1}{2}, \frac{3}{2}, \cdots, n-\frac{1}{2}$ is the total angular momentum. For each $j$, there are two degenerate states with orbital angular momentum $l=l_{ \pm}=j \pm \frac{1}{2}$, for which we define $\lambda_{j_{ \pm}} \equiv l_{ \pm}-\delta_{j}$, with

$$
\delta_{j} \equiv j+\frac{1}{2}-\sqrt{\left(j+\frac{1}{2}\right)^{2}-\hat{\alpha}^{2}}=\hat{\alpha}^{2} /(2 j+1)+\mathcal{O}\left(\hat{\alpha}^{4}\right) .
$$

The radial quantum number $n_{r}$ is related to the others by $n_{r}+\lambda_{j_{ \pm}}+1=n_{r}+l_{ \pm}+1-\delta_{l}=n-\delta_{j}$. 


\section{Semiclassical quantization for finite-size nucleus}

According to Eq. (120), the energy (117) becomes imaginary for the $l=0$-state of spin-0 particle when $\hat{\alpha}>1 / 2$, and of a spin- $\frac{1}{2}$ particle, when $\hat{\alpha}>1$. This signalizes the crash of the electron into the Coulomb potential of a point-like positive nuclear charge. The result is, however, unphysical since nuclei always have a finite radius $r_{\mathrm{n}}$. We account for this by the approximation a uniform distribution of charge inside the nucleus [7], so that the potential is

$$
V(r)=- \begin{cases}\hbar c \hat{\alpha} / r, & r \geq r_{\mathrm{n}} \\ \hbar c \hat{\alpha} f\left(r / r_{\mathrm{n}}\right) / r_{\mathrm{n}}, & r \leq r_{\mathrm{n}}\end{cases}
$$

where $f(x) \equiv\left(3-x^{2}\right) / 2$. Now the Sommerfeld quantization condition for the eikonal (110) reads

$$
S(\mathcal{E})=S^{(1)}(\mathcal{E})+S^{(2)}(\mathcal{E}) \equiv \int_{\tilde{r}_{0}}^{r_{\mathrm{n}}} d r p_{r}^{(1)}+\int_{r_{\mathrm{n}}}^{r_{\mathrm{o}}} d r p_{r}^{(2)}=\pi \hbar\left(n_{r}+\frac{1}{2}\right),
$$

with the radial momenta in the different regions:

$$
\begin{aligned}
& p_{r}^{(1)}=\frac{1}{c} \sqrt{\left[\mathcal{E}+\hbar c \frac{\hat{\alpha}}{r_{\mathrm{n}}} f\left(\frac{r}{r_{\mathrm{n}}}\right)\right]^{2}-c^{2} \hbar^{2} \frac{\left(l+\frac{1}{2}\right)^{2}}{r^{2}}-m_{e}^{2} c^{4}} \\
& p_{r}^{(2)}=\frac{1}{c} \sqrt{\left[\mathcal{E}+\hbar c \frac{\hat{\alpha}}{r}\right]^{2}-c^{2} \hbar^{2} \frac{\left(l+\frac{1}{2}\right)^{2}}{r^{2}}-m_{e}^{2} c^{4}}
\end{aligned}
$$

Let us first calculate the eikonal $S^{(1)}(\mathcal{E})$ inside the nucleus. Expanding $p_{r}^{(1)}$ in powers of $r / r_{\mathrm{n}}$ keeping terms up to the order $\mathcal{O}\left[\left(r / r_{\mathrm{n}}\right)^{2}\right]$ we obtain

$$
\begin{aligned}
p_{r}^{(1)} & \approx \frac{1}{c}\left\{\left(\mathcal{E}+c \hbar \frac{3 \hat{\alpha}}{2 r_{\mathrm{n}}}\right)^{2}-m_{e}^{2} c^{4}-\frac{\hat{\alpha}}{r_{\mathrm{n}}}\left(\mathcal{E}+c \hbar \frac{3 \hat{\alpha}}{2 r_{\mathrm{n}}}\right)\left(\frac{r}{r_{\mathrm{n}}}\right)^{2}-c^{2} \hbar^{2} \frac{\left(l+\frac{1}{2}\right)^{2}}{r^{2}}\right\}^{1 / 2} \\
& \approx \frac{1}{c}\left[\left(\mathcal{E}+c \hbar \frac{3 \hat{\alpha}}{2 r_{\mathrm{n}}}\right)^{2}-m_{e}^{2} c^{4}\right]^{1 / 2} \frac{1}{r}\left(r^{2}-\tilde{r}_{0}^{2}\right)^{1 / 2}
\end{aligned}
$$

where

$$
\tilde{r}_{0}=c \hbar\left[\frac{\left(l+\frac{1}{2}\right)^{2}}{\left(\mathcal{E}+c \hbar \frac{3 \hat{\alpha}}{2 r_{\mathrm{n}}}\right)^{2}-m_{e}^{2} c^{4}}\right]^{1 / 2} \approx \frac{2}{3 \hat{\alpha}}\left(l+\frac{1}{2}\right) r_{\mathrm{n}} .
$$

The approximation is good for $r_{\mathrm{n}} / \lambda_{C} \ll 1$, which is assured as long as

$$
|\mathcal{E}| \ll \hbar c \frac{\hat{\alpha}}{r_{\mathrm{n}}}, \quad \text { and } \quad \Lambda_{l}^{2} \equiv-\left(\lambda_{l}+\frac{1}{2}\right)^{2}=\hat{\alpha}^{2}-\left(l+\frac{1}{2}\right)^{2}>0 .
$$

The second inequality determines a maximum value of the angular-momentum $l$ for a given $\hat{\alpha}$. Inserting the approximate value (126) into (125), and the associated $p_{r}^{(1)}$ into (122), we obtain the 
$r<r_{\mathrm{n}}$-part of the eikonal

$$
\begin{aligned}
S^{(1)}(\mathcal{E}) & \approx m_{e} c\left[\left(r^{2}-\tilde{r}_{0}^{2}\right)^{1 / 2}+r_{\mathrm{n}}\left(l+\frac{1}{2}\right) \arcsin \left(\frac{\tilde{r}_{0}}{r}\right)\right]_{\tilde{r}_{0}}^{r_{\mathrm{n}}} \\
& =\hbar\left(\frac{r_{n}}{\lambda_{C}}\right)\left\{\left[1-\left(\frac{2 l+1}{3 \hat{\alpha}}\right)^{2}\right]^{1 / 2}+\left(l+\frac{1}{2}\right)\left[\arcsin \left(\frac{2 l+1}{3 \hat{\alpha}}\right)-\frac{\pi}{2}\right]\right\} .
\end{aligned}
$$

We now calculate the eikonal $S^{(2)}(\mathcal{E})$ outside the nucleus in Eq. (122). We begin with the negative-energy regime $-m_{e} c^{2}<\mathcal{E}<0$, where Eq. (127) is satisfied so that Eq. (112) gives $r_{\mathrm{o}} \gg r_{\mathrm{n}}$, and unphysical turning points $r_{\mathrm{i}}<0$ and $\left|r_{\mathrm{i}}\right|>r_{\mathrm{o}}$. Using Eq. (113) for $p_{r}^{(2)}(r)$ and integrating it over $r_{\mathrm{n}}<r<r_{\mathrm{o}}$ we find

$$
\begin{aligned}
& S^{(2)}(\mathcal{E}) \equiv \int_{r_{\mathrm{n}}}^{r_{\mathrm{o}}} d r p_{r}^{(2)}=\frac{1}{c} \sqrt{m_{e}^{2} c^{4}-\mathcal{E}^{2}} \\
& \times\left[R-\left(-r_{\mathrm{o}} r_{\mathrm{i}}\right)^{1 / 2} \ln \frac{2\left(-r_{\mathrm{o}} r_{\mathrm{i}}\right)+\left(r_{\mathrm{o}}+r_{\mathrm{i}}\right) r+2\left(-r_{\mathrm{o}} r_{\mathrm{i}}\right)^{1 / 2} R}{r}-\frac{r_{\mathrm{o}}+r_{\mathrm{i}}}{2} \arcsin \frac{\left(r_{\mathrm{o}}+r_{\mathrm{i}}\right)-2 r}{\left(r_{\mathrm{o}}-r_{\mathrm{i}}\right)}\right]_{r_{\mathrm{n}}}^{r_{\mathrm{o}}},
\end{aligned}
$$

where $R(r)=\sqrt{\left(r_{\mathrm{o}}-r\right)\left(r-r_{\mathrm{i}}\right)}$, and

$$
r_{\mathrm{o}}-r_{\mathrm{i}}=2 c \hbar \frac{\sqrt{\hat{\alpha}^{2} \mathcal{E}^{2}+\left(\lambda_{l}+\frac{1}{2}\right)^{2}\left(\mathcal{E}^{2}-m_{e}^{2} c^{4}\right)}}{m_{e}^{2} c^{4}-\mathcal{E}^{2}} .
$$

Under the conditions $r_{\mathrm{n}} / \lambda_{C} \ll 1$ and $r_{\mathrm{n}} /\left|r_{o, i}\right| \ll 1$, Eq. (129) becomes approximately

$$
S^{(2)}(\mathcal{E}) \approx \hbar\left[\frac{\hat{\alpha} \mathcal{E}}{\sqrt{m_{e}^{2} c^{4}-\mathcal{E}^{2}}}\left(\frac{\pi}{2}+\arcsin \frac{\hat{\alpha} \mathcal{E}}{\chi_{l}}\right)-\Lambda_{l}\left(1+\ln \frac{r_{\mathrm{n}} \chi_{l}}{c \hbar \Lambda_{l}^{2}}\right)\right],
$$

where

$$
\chi_{l} \equiv \sqrt{\Lambda_{l}^{2}\left(m_{e}^{2} c^{4}-\mathcal{E}^{2}\right)+\hat{\alpha}^{2} \mathcal{E}^{2}}
$$

For $r_{\mathrm{n}} \ll \lambda_{C}$, the first eikonal part $S^{(1)}(\mathcal{E})$ of Eq. (128) is negligible compared with the second part $S^{(2)}(\mathcal{E})$ of Eq. (131). Thus can simply do the calculation with a Coulomb potential cut off at $r=r_{\mathrm{n}}$. The charge distribution inside nucleus is irrelevant here.

For negative energies $\mathcal{E}$ close to zero, we approximate

$$
S^{(2)}(\mathcal{E}) \approx \hbar\left[\frac{1}{2 \Lambda_{l}}\left(\hat{\alpha}^{2}+\Lambda_{l}^{2}\right)\left(\frac{\mathcal{E}}{m_{e} c^{2}}\right)^{2}+\frac{\pi \hat{\alpha}}{2}\left(\frac{\mathcal{E}}{m_{e} c^{2}}\right)-\Lambda_{l}\left(1+\ln \frac{r_{\mathrm{n}}}{\lambda_{C} \Lambda_{l}}\right)\right],
$$

and the quantization rule (122) yields the energy levels

$$
\mathcal{E}_{n l} \approx-m_{e} c^{2} \frac{\pi \hat{\alpha} \Lambda_{l}+\sqrt{\left(\pi \hat{\alpha} \Lambda_{l}\right)^{2}+8\left(\hat{\alpha}^{2}+\Lambda_{l}^{2}\right)\left[\pi\left(n_{r}+\frac{1}{2}\right)+\Lambda_{l}\left(1+\ln \frac{r_{n}}{\lambda_{C} \Lambda_{l}}\right)\right]}}{2\left(\hat{\alpha}^{2}+\Lambda_{l}^{2}\right)} .
$$

For negative energies $\mathcal{E}$ close to $-m_{e} c^{2}$, expression (131) simplifies to

$$
S^{(2)}(\mathcal{E}) \approx \hbar\left[\left(\frac{\Lambda_{l}}{2}-\frac{\Lambda_{l}^{3}}{6 \hat{\alpha}^{2}}\right) \eta^{2}-\Lambda_{l}\left(2+\ln \frac{r_{\mathrm{n}} \hat{\alpha}}{\lambda_{C} \Lambda_{l}^{2}}\right)\right],
$$


where $\eta^{2} \equiv\left(m_{e}^{2} c^{4}-\mathcal{E}^{2}\right) /\left(m_{e} c^{2}\right)^{2}$, so that the quantization rule (122) gives

$$
\mathcal{E}_{n l} \approx-m_{e} c^{2}\left[1-6 \hat{\alpha}^{2} \frac{\pi\left(n_{r}+\frac{1}{2}\right)+\Lambda_{l}\left(2+\ln \frac{r_{\mathrm{n}} \hat{\alpha}}{\lambda_{C} \Lambda_{l}^{2}}\right)}{3 \hat{\alpha}^{2} \Lambda_{l}-\Lambda_{l}^{3}}\right]^{1 / 2} .
$$

The critical value $\hat{\alpha}_{c}(l)$ can be obtained from Eq. (122), and from (135) for $\eta^{2}=0$,

$$
\Lambda_{l}^{2}=\hat{\alpha}_{c}^{2}-\left(l+\frac{1}{2}\right)^{2} \approx \pi^{2}\left[\frac{n_{r}+\frac{1}{2}}{2+\ln \frac{r_{\mathrm{n}} \hat{\alpha}}{\lambda_{C} \Lambda_{l}^{2}}}\right]^{2} .
$$

Consider now the negative-energy regime below $-m_{e} c^{2}$ under the assumption (127), so that Eq. (112) yields $r_{\mathrm{o}} \gg r_{\mathrm{n}}$, and unphysical zeros at $0<r_{\mathrm{o}}<r_{\mathrm{i}}$. By writing $p_{r}$ in Eq. (111) by analogy with in (113) as

$$
p_{r}=\frac{1}{c} \sqrt{\mathcal{E}^{2}-m_{e}^{2} c^{4}} \frac{1}{r} \sqrt{\left(r_{\mathrm{o}}-r\right)\left(r_{\mathrm{i}}-r\right)}
$$

we can perform the second integral in (122) and obtain

$$
\begin{aligned}
& S^{(2)}(\mathcal{E}) \equiv \int_{r_{\mathrm{n}}}^{r_{\mathrm{o}}} d r p_{r}=\frac{1}{c} \sqrt{\mathcal{E}^{2}-m_{e}^{2} c^{4}} \\
& \quad \times\left[\bar{R}-\left(r_{\mathrm{o}} r_{\mathrm{i}}\right)^{1 / 2} \ln \frac{2\left(r_{\mathrm{o}} r_{\mathrm{i}}\right)-\left(r_{\mathrm{o}}+r_{\mathrm{i}}\right) r+2\left(r_{\mathrm{o}} r_{\mathrm{i}}\right)^{1 / 2} \bar{R}}{r}-\frac{r_{\mathrm{o}}+r_{\mathrm{i}}}{2} \ln \left[2 \bar{R}+2 r-\left(r_{\mathrm{o}}+r_{\mathrm{i}}\right)\right]\right]_{r_{\mathrm{n}}}^{r_{\mathrm{o}}},
\end{aligned}
$$

where $\bar{R}(r)=\sqrt{\left(r_{\mathrm{o}}-r\right)\left(r_{\mathrm{i}}-r\right)}$. Under the conditions $r_{\mathrm{n}} / \lambda_{C} \ll 1$ and $r_{\mathrm{n}} / r_{o, i} \ll 1$, we obtain

$$
S^{(2)}(\mathcal{E}) \approx \hbar\left[\frac{\hat{\alpha}|\mathcal{E}|}{\sqrt{\mathcal{E}^{2}-m_{e}^{2} c^{4}}} \ln \left(\Lambda_{l} \frac{\sqrt{\mathcal{E}^{2}-m_{e}^{2} c^{4}}}{\hat{\alpha}|\mathcal{E}|}-1\right)-\Lambda_{l}\left(1+\ln \frac{r_{\mathrm{n}} \chi_{l}}{c \hbar \Lambda_{l}^{2}}\right)\right] .
$$

Assuming $\hbar c \hat{\alpha} / r_{\mathrm{n}} \gg|\mathcal{E}| \gg m_{e} c^{2}$, we expand $S^{(2)}(\mathcal{E})$ in terms of $m_{e} c^{2} /|\mathcal{E}|$ and the leading order is

$$
S^{(2)}(\mathcal{E}) \approx \hbar \Lambda_{l}\left[\ln \frac{m_{e} c^{2}}{|\mathcal{E}|}+\frac{\hat{\alpha}}{\Lambda_{l}} \ln \left(\frac{\Lambda_{l}}{\hat{\alpha}}-1\right)-1-\ln \frac{r_{\mathrm{n}}}{\lambda_{C} \Lambda_{l}^{2}}-\ln \left(\hat{\alpha}^{2}-\Lambda_{l}^{2}\right)\right],
$$

and the quantization rule (122) yields energy-levels:

$$
\mathcal{E}_{n l} \approx-m_{e} c^{2} \frac{\lambda_{C}}{r_{\mathrm{n}}}\left[\frac{\Lambda_{l}^{2}\left(\frac{\Lambda_{l}}{\hat{\alpha}}-1\right)^{\frac{\hat{\alpha}}{\Lambda_{l}}}}{\hat{\alpha}^{2}-\Lambda_{l}^{2}}\right] \exp \left[-\frac{\pi}{\Lambda_{l}}\left(n_{r}+\frac{1}{2}\right)-1\right] .
$$

As for a spin- $\frac{1}{2}$ particle, the critical value $\hat{\alpha}_{c}(j)$ and energy-spectra $\mathcal{E}_{n j}$ are obtained from the critical value (137) and energy-spectra (136) and (142) by the following replacement:

$$
\Lambda_{l}^{2}=\hat{\alpha}^{2}-\left(l+\frac{1}{2}\right)^{2} \Rightarrow \Lambda_{j}^{2}=\hat{\alpha}^{2}-\left(j+\frac{1}{2}\right)^{2} .
$$

As before, for each $j$ there are two degenerate levels of orbit angular momentum $l=l_{ \pm}=j \pm \frac{1}{2}$. 


\section{WKB transmission probability}

The Schrödinger equation Eq. (106) can be written as

$$
\left[c^{2} \hbar^{2} \frac{d^{2}}{d r^{2}}+c^{2} p_{r}^{2}(r)\right] r R_{\mathcal{E}, l}(r)=0,
$$

where

$$
c^{2} p_{r}^{2}=[\mathcal{E}-V(r)]^{2}-c^{2} \hbar^{2} \frac{\left(l+\frac{1}{2}\right)^{2}}{r^{2}}-m_{e}^{2} c^{4}
$$

which looks like the Klein-Gordon equation (6) in one dimension along the $r$-axis. As before, we assume the nuclear radius $r_{\mathrm{n}}$ to be much smaller than the Compton wavelength $\ll \lambda_{C}$, Outside the nucleus, $V(r)$ is the Coulomb potential. From th condition $p_{r}=0$ we calculate the classical turning points $r_{\mathrm{n}} \ll r_{+}<r_{-}$, by analogy with Eq. (44). For a give energy $\mathcal{E}<-m_{e} c^{2}$, we have three regions (see Fig. 6):

(i) $r_{-}<r$ and $\mathcal{E}<\mathcal{E}_{-}$,

(ii) $r_{+}<r<r_{-}$and $\mathcal{E}_{-}<\mathcal{E}<\mathcal{E}_{+}$,

(iii) $r<r_{+}$and $\mathcal{E}>\mathcal{E}_{+}$.

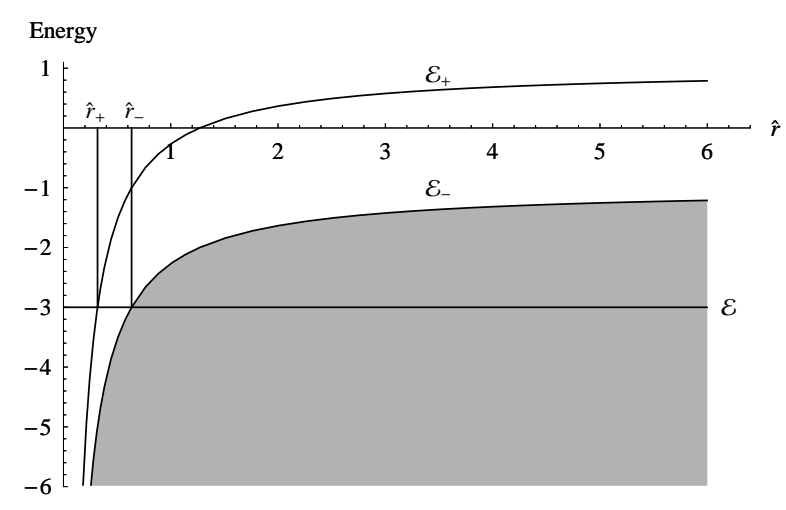

FIG. 6: For a radial Coulomb field, the positive and negative-energy spectra $\mathcal{E}_{ \pm}(r)$ of Eq. (2) are plotted as a function of $\hat{r}=r / \lambda_{C}$. They are found by solving condition $p_{r}=0$ at $l=0$ and $\hat{\alpha}=1.27(Z=174)$.

Starting (i) where $p_{r}^{2}>0$, Eq. (144) has two independent solutions corresponding to $p_{r}<0$ (incident wave) and $p_{r}>0$ (reflected wave),

$$
R_{\mathcal{E}, l}=\frac{\mathcal{C}_{+}^{r}}{\left(p_{r}\right)^{1 / 2}} \frac{1}{r} \exp \left[\frac{i}{\hbar} \int^{r} p_{r} d r\right]+\frac{\mathcal{C}_{-}^{r}}{\left(p_{r}\right)^{1 / 2}} \frac{1}{r} \exp \left[-\frac{i}{\hbar} \int^{r} p_{r} d r\right]
$$


where $\mathcal{C}_{ \pm}^{r}=e^{ \pm i \pi / 4} \mathcal{C}^{r} / 2$. The corresponding solution in the region (ii) is given by

$$
\frac{\mathcal{C}^{r}}{2\left(\kappa_{r}\right)^{1 / 2}} \frac{1}{r} \exp \left[-\frac{1}{\hbar} \int_{r}^{r_{-}} \kappa_{r} d r\right]+\frac{\overline{\mathcal{C}}^{r}}{2\left(\kappa_{r}\right)^{1 / 2}} \frac{1}{r} \exp \left[\frac{1}{\hbar} \int_{r}^{r_{-}} \kappa_{r} d r\right]
$$

where $\kappa_{r}=-i p_{r}$ and $p_{r}^{2}<0$. For a purely incident wave, only the second term in $R_{\mathcal{E}^{-}, l}$ is present, with an incident flux density at $r=r_{-}$:

$$
j_{r} \equiv \frac{\hbar}{2 m_{e} i}\left[\phi_{r}^{*} \hat{\partial}_{r} \phi_{r}-\left(\hat{\partial}_{r} \phi_{r}^{*}\right) \phi_{r}\right]=-\frac{p_{r}}{m_{e}} \phi_{r}^{*} \phi_{r}=-\frac{\left|\mathcal{C}_{-}^{r}\right|^{2}}{m_{e} r_{-}^{2}}
$$

where $\phi_{r}=r R_{\mathcal{E}, l}^{-}$and $\hat{\partial}_{r}=(1 / r)(\partial / \partial r) r$. The superscript - indicates the inward flux.

We consider bound states of energy $\mathcal{E}<-m_{e} c^{2}$ confined within the region $\left(\tilde{r}_{0}, r_{+}\right)$, where $\tilde{r}_{0}<r_{\mathrm{n}}$ is another classical turning point of positive-energy branch $\mathcal{E}_{+}$inside the nucleus, in addition to $r_{+}$. Its value is obtained from Eq. (145) for $p_{r}=0$ :

$$
\mathcal{E}-V\left(\tilde{r}_{0}\right)=+\sqrt{c^{2} \hbar^{2} \frac{\left(l+\frac{1}{2}\right)^{2}}{\tilde{r}_{0}^{2}}+m_{e}^{2} c^{4}} .
$$

By analogy with Eq. (89), the semi-classical wave function of bound states is,

$$
R_{\mathcal{E}, l}^{\mathrm{bs}}=\frac{\mathcal{B}^{r}}{p_{r}^{1 / 2}} \frac{1}{r} \cos \left[\frac{1}{\hbar} \int_{\tilde{r}_{0}}^{r} p_{r} d r-\frac{\pi}{4}\right]
$$

satisfying Sommerfeld's quantization rule

$$
\frac{1}{\hbar} \int_{\tilde{r}_{0}}^{r_{+}} p_{r} d r=\pi\left(n_{r}+\frac{1}{2}\right), \quad n_{r}=0,1,2, \cdots
$$

For each $n_{r}=0,1,1 \ldots$, the solutions yield the discrete energies $\mathcal{E}_{n l}$ with the principal quantum number $n \equiv n_{r}+l+1$, where the angular momentum $l$ can take the values $l=0, \ldots, n$. The continuity of wave functions (147) and (150) at the classical point $r_{+}$leads to

$$
\mathcal{B}^{r}=\sqrt{2} \mathcal{C}_{-}^{r} e^{-i \pi n_{r}} \exp \left[-\frac{1}{\hbar} \int_{r_{+}}^{r_{-}} \kappa_{r} d r\right] .
$$

Assuming the bound states $R_{\mathcal{E}, l}^{\text {bs }}$ be unoccupied, the transmitted flux to these states at the classical turning point $r_{+}$is

$$
\left.\frac{\hbar}{m_{e}} R_{\mathcal{E}, l}^{\mathrm{bs}} \hat{\partial}_{r}\left[R_{\mathcal{E}, l}^{\mathrm{bs}}\right]^{*}\right|_{r \rightarrow r_{+}}=-\frac{\left|\mathcal{B}^{r}\right|^{2}}{2 m_{e} r_{+}^{2}}=-\frac{\left|\mathcal{C}_{-}^{r}\right|^{2}}{m_{e} r_{+}^{2}} \exp \left[-\frac{2}{\hbar} \int_{r_{+}}^{r_{-}} \kappa_{r} d r\right] .
$$

From Eqs. (148), (153), and (20) we obtain the transmission probability of an electron to tunnel into the bound state:

$$
W_{\mathrm{WKB}}(\mathcal{E}, l)=\frac{r_{-}^{2}}{r_{+}^{2}} \exp \left[-\frac{2}{\hbar} \int_{r_{+}}^{r_{-}} \kappa_{r} d r\right]
$$


Normalizing the incident flux density (148) at $r_{-}$we have

$$
j_{r} \equiv \sum_{l} j_{r}^{l}=\sum_{l} D_{s} v_{r}\left(r_{-}\right) \frac{(2 l+1)}{4 \pi r_{-}^{2}} \int \frac{d p_{r}}{2 \pi \hbar},
$$

and the rate of pair-production in the state with angular momentum $l$ becomes:

$$
\begin{aligned}
\frac{\Gamma_{\mathrm{WKB}}(\mathcal{E}, l)}{V_{\perp}} & =W_{\mathrm{WKB}}(\mathcal{E}, l) j_{r}^{l} \\
& =D_{s} v_{r}\left(r_{-}\right) \frac{(2 l+1)}{4 \pi r_{+}^{2}} \int \frac{d p_{r}}{2 \pi \hbar} \exp \left[-\frac{2}{\hbar} \int_{r_{+}}^{r_{-}} \kappa_{r} d r\right] \\
& =D_{s} \frac{(2 l+1)}{4 \pi r_{+}^{2}} \int \frac{d \mathcal{E}}{2 \pi \hbar} \exp \left[-\frac{2}{\hbar} \int_{r_{+}}^{r_{-}} \kappa_{r} d r\right]
\end{aligned}
$$

where $v_{r}\left(r_{-}\right)=\partial \mathcal{E} /\left.\partial p_{r}\right|_{r=r_{-}}$. This is evaluated further in the same way as Eqs. (96) and (97): the integral over $\mathcal{E}$ has only contributions from the bound state energies $\mathcal{E}=\mathcal{E}_{n l}$, so that $\int d \mathcal{E}$ is equal to $\omega_{n l} \hbar$, where $\omega_{n l}=\mathcal{E}_{n l} / \hbar$ is the frequency of the bound state. As a result, the sum over all Eq. (156) takes the form

$$
\frac{\Gamma_{\mathrm{WKB}}}{V_{\perp}}=D_{s} \sum_{n l} \frac{(2 l+1)}{4 \pi r_{+}^{2}} \frac{\omega_{n l}}{2 \pi} \exp \left[-\frac{2}{\hbar} \int_{r_{+}}^{r_{-}} \kappa_{r} d r\right] .
$$

\section{Sauter exponential factor in Coulomb potential}

For brevity we use natural units where $r$ is measured in units of the Compton wavelengths $\lambda_{C}=\hbar / m_{e} c$, and $\mathcal{E}_{n l}$ in units of $m_{e} c^{2}$. By setting $p_{r}=0$ in Eq. (111), we obtain the analog of Eq. (24):

$$
\frac{1}{r\left(l, \mathcal{E}_{n l} ; \zeta\right)}=\frac{\mathcal{E}_{n l} \hat{\alpha}+\zeta \sqrt{\left(l+\frac{1}{2}\right)^{2}\left(\mathcal{E}_{n l}^{2}-\zeta^{2}\right)+\hat{\alpha}^{2}}}{\left(l+\frac{1}{2}\right)^{2} \zeta^{2}-\hat{\alpha}^{2}} .
$$

The role of $p_{\perp}^{2}$ is now played by $\left(l+\frac{1}{2}\right)^{2} / r^{2}$. The classical turning point $r_{-}\left(\mathcal{E}_{n l}, l\right)$ where the negative-energy states can tunnel to the positive ones at $r_{+}\left(\mathcal{E}_{n l}, l\right)$ is obtained from Eq. (158) by inserting $\zeta= \pm 1$. We identify $r_{+} \equiv r_{\mathrm{o}}$ in previous sections. Since $r_{-}>r_{+}$, the electrons move inwards to neutralize the Coulomb potential, the positrons are pushed outwards to infinity. At the highest energy $\mathcal{E}_{n l}=-1$ where tunneling can occur, we find $r_{-}=+\infty$ and $r_{+}=\left[\hat{\alpha}^{2}-\left(l+\frac{1}{2}\right)^{2}\right] / 2 \hat{\alpha}$.

For each angular momentum $l$ and energy-level $\mathcal{E}_{n l}$, we obtain the Sauter exponential factor from the analogs of Eqs. (26) and (27):

$$
\exp \left[-\frac{2}{\hbar} \int_{r_{+}}^{r_{-}} \kappa_{r} d r\right]=\exp \left\{-\pi \frac{E_{c}}{E_{0}} G\left(l, \mathcal{E}_{n l}\right)\right\},
$$


and

$$
G\left(l, \mathcal{E}_{n l}\right) \equiv \frac{2}{\pi} \int_{-1}^{1} d \zeta\left[1+\frac{\left(l+\frac{1}{2}\right)^{2}}{r^{2}\left(l, \mathcal{E}_{n l} ; \zeta\right)}\right] \frac{\sqrt{1-\zeta^{2}}}{E\left(l, \mathcal{E}_{n l} ; \zeta\right) / E_{0}}
$$

Note that the bracket in the integral is the analog of the prefactor $\left[1+\left(c p_{\perp}\right)^{2} / m_{e}^{2} c^{4}\right]$ in (26). Here it can no longer be taken out of the integral. Instead, there is now another simplification. Since $E=\hat{\alpha} \hbar c / r^{2}$, the function $G\left(l, \mathcal{E}_{n l}\right)$ becomes

$$
G\left(l, \mathcal{E}_{n l}\right) \equiv \frac{2}{\pi} \int_{-1}^{1} d \zeta \sqrt{1-\zeta^{2}}\left[r^{2}\left(l, \mathcal{E}_{n l} ; \zeta\right)+\left(l+\frac{1}{2}\right)^{2}\right]
$$

and with $r(l, \mathcal{E} ; \zeta)$ from (158):

$$
G\left(l, \mathcal{E}_{n l}\right) \equiv \frac{2}{\pi} \int_{-1}^{1} d \zeta \sqrt{1-\zeta^{2}}\left\{\frac{\left[\left(l+\frac{1}{2}\right)^{2} \zeta^{2}-\hat{\alpha}^{2}\right]^{2}\left[\left(\mathcal{E}_{n l} \hat{\alpha}\right)^{2}+\zeta^{2}\left(\left(l+\frac{1}{2}\right)^{2}\left(\mathcal{E}_{n l}^{2}-\zeta^{2}\right)+\hat{\alpha}^{2}\right)\right]}{\left[\left(\mathcal{E}_{n l} \hat{\alpha}\right)^{2}-\zeta^{2}\left(\left(l+\frac{1}{2}\right)^{2}\left(\mathcal{E}_{n l}^{2}-\zeta^{2}\right)+\hat{\alpha}^{2}\right)\right]^{2}}+\left(l+\frac{1}{2}\right)^{2}\right\}
$$

The result of this integral is surprisingly simple remembering that $r_{ \pm}$are given by $r_{ \pm}\left(\mathcal{E}_{n l} ; l\right)$ of Eq. (158) for $\zeta= \pm 1$ :

$$
G\left(l, \mathcal{E}_{n l}\right)=2\left(l+\frac{1}{2}\right)^{2}\left(\mathcal{E}_{n l}^{2}-\left|\mathcal{E}_{n l}\right| \sqrt{\mathcal{E}_{n l}^{2}-1}\right)+2 \hat{\alpha}^{2}\left(\frac{\sqrt{\mathcal{E}_{n l}^{2}-1}}{\left|\mathcal{E}_{n l}\right|}-1\right)
$$

valid for $\mathcal{E}_{n l}<-1$ and $\hat{\alpha} \geq \hat{\alpha}_{c}(l)$. Inserting this into the Sauter exponential factor (159), and this further into (157), we obtain the rate of filling the empty bound state levels around the Coulomb potential of the nucleus. The sum over $l$ extends to the largest value permitted by Eq. (127) and $\hat{\alpha}_{c}$ of (137).

The result for a spin- $\frac{1}{2}$ particle can be obtained by replacing $\mathcal{E}_{n l} \Rightarrow \mathcal{E}_{n j}$ in Eqs. (158) and (163), and

$$
\left(l+\frac{1}{2}\right)^{2} \Rightarrow\left(j+\frac{1}{2}\right)\left(j \pm 1+\frac{1}{2}\right)
$$

where $j \pm 1$ respectively corresponds the state of parity $(-)^{l_{ \pm}}$with orbital angular momentum $l_{ \pm}=j \pm \frac{1}{2}$.

\section{SUMMARY AND REMARKS}

By studying the process of electron-positron pair production from the vacuum by a nonuniform electric field as a quantum tunneling phenomenon we have derived in semiclassical approximation the general rate formulas (38) and (39). They consist of a Boltzmann-like tunneling exponential, and a pre-exponential factor, and are applicable to any system where the field strength points mainly in one direction and varies only along this direction. The formulas require the evaluation of 
the functions $G(0, \mathcal{E})$ of Eq. (32) and $G_{\delta}(0, \mathcal{E})$ of Eq. (33). This has been done for several different field configurations.

For electrons arriving by tunneling in a bound state of a harmonic electric potential, the general expressions are given by Eqs. (97) and (157) as functions of the frequencies $\omega_{n}$ of the bound states. The discrete energy levels $\mathcal{E}_{n}$ at fixed $p_{\perp}$ are found from the eikonal and Sommerfeld quantization. For fermions, the expression for pair-production rate should be multiplied by the Pauli-blocking factor for the rate which makes it zero if the bound state $\mathcal{E}_{n}$ is occupied.

In the Coulomb electric field $E(r)=e Z / r^{2}$ of a nucleus with finite radius $r_{\mathrm{n}} \ll \lambda_{C}$, we have given first the semiclassical energy-levels $\mathcal{E}_{n l}$ in Eqs. (117), (134), (136), (142), and the formulas for the associated pair production rate in (157), (159), (160) for $\mathcal{E}_{n l} \leq-m_{e} c^{2}$. The critical value $\hat{\alpha}_{c} \equiv Z_{c} \alpha$ is found from Eq. (137) as a function of the principal quantum number $n$ and the angular momentum $l$, which agree with the one found in Refs. [7, 39] the $n=1$-state $1 S_{\frac{1}{2}}$.

The number of energy-levels $\mathcal{E}_{n l} \leq-m_{e} c^{2}$ that are able to accommodate electrons produced from the vacuum is limited, and pair production ceases when all these levels are fully occupied even if the electric field is overcritical.

[1] F. Sauter, Z. Phys. 69 (1931) 742 (paper is in German, English translation can be downloaded from http://www.physik.fu-berlin.de/ ${ }^{k}$ leinert/files/sautere.pdf).

[2] W. Heisenberg and H. Euler, Z. Phys. 98 (1936) 714 (paper is in German, English translation can be

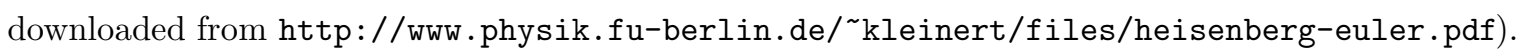

[3] J. Schwinger, Phys. Rev. 82 (1951) 664; Phys. Rev. 93 (1954) 615; Phys. Rev. 94 (1954) 1362.

[4] G. V. Dunne, arXiv:hep-th/0406216, in Ian Kogan Memorial Collection, "From Fields to Strings: Circumnavigating Theoretical Physics".

[5] R. Ruffini, G. V. Vereshchagin and S.-S. Xue, a review article on "Electron-positron pair production, annihilation and oscillation in external electric fields" (to be submitted to Phys. Rep.).

[6] Review article: A. Ringwald, Phys. Lett. B510 (2001) 107, and talk presented at the "Workshop on Quantum Aspects of Beam Physics", Hiroshima, Japan, January 2003 (references there in).

[7] Review article: Ya. B. Zel'dovich and V. S. Popov, Uspekhi Fiz. Nauk 103 (1971) 403, [Sov. Phys. Uspekhi 14 (1972) 673] (references there in).

[8] Review article: W. Greiner, B. Müller and J. Rafelski, "Quantum Electrodynamics of Strong Fields" Monograph in Physics, ISBN 3-540-13404-2 Springer-Verlag Berlin Heidelberg (1985) (references there in).

[9] T. Damour and R. Ruffini, Phys. Rev. Lett. 35 (1975) 463;

G. Preparata, R. Ruffini and S.-S. Xue, Astron. Astroph. Lett. 337 (1998) L3., and J. Korean 
Phys.Soc. 42 (2003) S99-S104 (astro-ph/0204080);

R. Ruffini, L. Vitagliano and S.-S. Xue, Phys. Lett. B573 (2003) 33;

M. Rotondo, R. Ruffini and S.-S. Xue, Int. Journal of Modern Phys. D Vol. 16, No. 1 (2007) 1-9.

[10] R. Ruffini, L. Vitagliano and S.-S. Xue, Phys. Lett. B559 (2003) 12;

R. Ruffini, G. V. Vereshchagin and S.-S. Xue, Phys. Lett. A371 (2007) 399-405, arXiv:0706.4363 (references of plasma oscillation there in).

[11] H. Kleinert, Path Integrals in Quantum Mechanics, Statistice, Polymer Physics, and Financial Markets, 4th ed., World Scientific, Singapore 2006 (downloadable from http://www.physik.fu-berlin.de/ ${ }^{\sim}$ kleinert/b5). See in particular Chapter 4.

[12] C. M. Martin and D. Vautherin, Phys. Rev. D38 (1988) 3593; Phys. Rev. D40 (1989) 1667.

[13] T.N. Tomaras and N.C. Tsamis and R.P. Woodard, Phys. Rev. D62 (2000) 125005;// J. Avan, H.M. Fried and Y. Gabellini, Phys. Rev. D67 (2003) 016003.

[14] N. B. Narozhnyi and A. I. Nikishov, Yad. Fiz. 11 (1970) 1072 [Sov. J. Nucl. Phys. 11 (1970) 596].

[15] Review article: J. Rafelski, L.P. Fulcher and A. Klein, Phys. Reps. 38 (1978) 227.

[16] E. Brezin and C. Itzykson, Phys. Rev. D2 (1970) 1191.

[17] V. S. Popov, Zh. Eksp. Theor. Fiz. 61 (1971) 1334 [Sov. Phys. JETP 34 (1972) 709].

[18] Ren-Chuan Wang and Cheuk-Yin Wong, Phys. Rev. D38 (1988) 248.

[19] I. B. Khriplovich, Il Nuovo Cimento B115, (2000) 761.

[20] S. P. Kim and D. N. Page, Phys. Rev. D65 (2002) 105002;

S. P. Kim and D. N. Page, Phys. Rev. D73 (2006) 065020.

[21] S. P. Kim and D. N. Page, Phys. Rev. D75 (2007) 045013.

[22] G. Schubert, Phys. Rep. 355 (2001) 73.

[23] G. V. Dunne and C. Schubert, Phys. Rev. D72 (2005) 105004.

[24] G. V. Dunne, Q.-H. Wang, H. Gies and C. Schubert, Phys. Rev. D73 (2006) 065028.

[25] H. Gies and K. Langfeld, Nucl. Phys. B613 (2001) 353, Int. J. Mod. Phys. A17 (2002) 966;

K. Langfeld, L. Moyaerts and H. Gies, Nucl. Phys. B646 (2002) 158, J. High Energy Phys. 0306 (2003) 018.

[26] H. Gies and K. Klingmüller, Phys. Rev. D72 (2005) 065001.

[27] G. V. Dunne and Q.-H. Wang, Phys. Rev. D74 (2006) 065015.

[28] P. A. M. Dirac, Proc. of the Cambridge Philos. Soc. 26 (1930) 361; Proc. of the Roy. Soc. of London 126 (1930) 360; see also J. R. Oppenheimer, Phys. Rev. 35 (1930) 562; Phys. Rev. 35 (1930) 939.

[29] P. A. M. Dirac, Rep. at Inst. of Phys. Solvay (1933) 203.

[30] L. D. Landau and E. M. Lifshitz, Non-Relativistic Quantum Mechanics, Pergamon Press, Oxford, 1975.

[31] H. Kleinert, Path Integrals in Quantum Mechanics, Statistics, Polymer Physics, and Financial Markets, 4th ed., World Scientific, Singapore 2006 (downloadable from http://www.physik.fu-berlin.de/ kleinert/b5). See in particular Chapter 4.

[32] By setting $D_{s}$ equal to 1 one can obtain the tunneling result also for spin-0 particles although the Dirac 
picture is no longer applicable.

[33] Note that omitting the $z$-integral in the rate formula (38) does not justify calling the result a "local production rate", as done in the abstract of Ref. [26]. The result is always nonlocal and depends on all gradients of the electric field.

[34] See Eq. (63) of Ref. 23], and replace there $\tilde{\gamma} \rightarrow 1 / \sigma$. It agrees also with Eq. (4.7) of the later paper by Dunne et al. 24] apart from a factor $1 / 2$.

[35] See Section 2.2 in the textbook [31].

[36] This treatment is analogous to that of the translational degree of freedom in instanton calculations in Section 17.3.1 of 31] [see in particular Eq. (17.112)].

[37] R.E. Langer, Phys. Rev. 51, 669 (1937); W.H. Furry, Phys. Rev. 71, 360 (1947); P.M. Morse and H. Feshbach, Methods of Theoretical Physics, McGraw-Hill, 1953, pp. 1092ff. See also Eq. (14.83) in the textbook [31]

[38] This term will be examined within the original coordinate as a purely relativistic effect in a coming paper.

[39] V. S. Popov, Yad. Fiz. 12 (1970) 429 [Sov. J. Nucl. Phys. 12 (1971) 235], Zh. Eksp. Theor Fiz. 60 (1971) 1228 [Sov. Phys. JETP 33 (1971) 665]. 